\title{
Multiple epigenetic layers accompany the spatial distribution of ribosomal genes in Arabidopsis
}

\author{
*Konstantin O. Kutashev 1,2, *Michal Franek ${ }^{1}$, Klev Diamanti ${ }^{3,4}$, Jan Komorowski ${ }^{4,5}$, \\ Marie Olšinová ${ }^{6}$, Martina Dvořáčková ${ }^{1}$ \\ ${ }^{1}$ Mendel Centre for Plant Genomics and Proteomics, CEITEC, Masaryk University, Kamenice 5, 62500, \\ Brno, Czech Republic \\ ${ }^{2}$ Laboratory of Functional Genomics and Proteomics, National Centre for Biomolecular Research, Faculty \\ of Science, Masaryk University, Kotlářská 2, 61137, Brno, Czech Republic \\ ${ }^{3}$ Department of Cell and Molecular Biology, Uppsala University, Uppsala 751 24, Sweden \\ ${ }^{4}$ Department of Immunology, Genetics and Pathology, Uppsala University, Uppsala 751 08, Sweden \\ ${ }^{5}$ Institute of Computer Science, Polish Academy of Sciences, Warsaw 012-48, Poland \\ ${ }^{6}$ BioCEV Imaging Methods Core Facility, Průmyslová 595, 25250 Vestec, Czech Republic \\ * these authors contributed equally to the work
}

Corresponding author: Martina Dvořáčková

Email: martina.dvorackova@ ceitec.muni.cz; ORCID: MD 0000-0001-5998-6159; tel:+420549495601

Running title: Epigenetic regulation of ribosomal genes

Keywords: rDNA, nucleolar foci, histone variants, ChIP, super-resolution microscopy

Final character count: $\sim 60000$ (including title page, references, figure legends) 


\begin{abstract}
45S ribosomal genes in A. thaliana (rDNA) are located in tandem arrays on termini of chromosomes 2 and 4 (NOR2 and NOR4) and encode rRNA, crucial structural elements of the ribosome. Inactive rDNA genes accumulate in the condensed chromocenters in the nucleus and at the nucleolar periphery, while nucleolus delimits the active genes. We show that a subset of nucleolar rDNA assembles into condensed foci marked by $\mathrm{H} 3.1$ and $\mathrm{H} 3.3$ histones and that progressive rDNA condensation is connected with rDNA transcriptional activity, cell ploidy and rDNA copy number. Interestingly, some nucleolar foci are reminiscent of perinucleolar chromocenters, containing the NOR4 region. We further demonstrate that rDNA promoter is a key regulatory region of the rDNA repeat and describe large involvement of repressive epigenetic mark $\mathrm{H} 3 \mathrm{~K} 9 \mathrm{me} 2$ and $\mathrm{H} 2 \mathrm{~A} . \mathrm{W}$ histone variant in rDNA activity regulation. In addition, we found euchromatic $\mathrm{H} 3.3$ histone enrichment at the rDNA transcription start site in actively dividing tissues, despite its accumulation in nucleolar foci containing condensed rDNA repeats.
\end{abstract}

\title{
Introduction
}

Ribosomal rRNA genes (rDNA, 45S rDNA) belong to the group of essential house-keeping genes, encoding key components of the ribosome $-18 \mathrm{~S}, 5.8 \mathrm{~S}$ and $25 \mathrm{~S}$ rRNA - required for protein production. Ribosomal genes in A. thaliana are organized into large clusters located at chromosome termini, containing from several hundreds to thousands of rDNA repeats. Such regions represent challenging targets for investigation. In A. thaliana two rDNA loci (3-5 MBp each) can be found at the short arms of chromosomes 2 and 4 (referred to as NOR2 and NOR4) where 500-700 copies of the $10 \mathrm{~kb}$ long rDNA repeat occur (Copenhaver et al, 1995; Pruitt \& Meyerowitz, 1986). Individual NORs differ in their transcriptional activity: in Col-0 ecotype, NOR2 genes are inactive and show nucleoplasmic localisation (Borysko \& Bang, 1951; Pontvianne et al, 2013; Shaw \& Jordan, 1995), while rDNA at NOR4 is transcribed and associates to the nucleolus as one of the nucleolus associated domains (Chandrasekhara et al, 2016). The separation of the two NORs is incomplete: the rDNA bearing chromosomes tend to fuse more often than other genomic loci (Berr $\&$ Schubert, 2007), complicating the analysis of individual rDNA fractions. In addition, transcribed rDNA genes at NOR4 represent a minority of rDNA, occurring in a largely decondensed and fragile form, even escaping detection (Huang et al, 2008). Regardless of the numerous attempts to find suitable methods to distinguish between active and inactive rDNA units (e.g. determination of 3 'external transcribed spacer (ETS) variants by PCR or back crossing) (Chandrasekhara et al, 2016; Pontvianne et al, 2010), microscopy remains the method of choice. It is because active rDNA of NOR4 is spatially delimited by the nucleolus, emerging as a consequence of rDNA transcription by RNA polymerase I and represents one of the most prominent nuclear structures in plant nuclei (McKeown \& Shaw, 2009; Stepinski, 2014).

Nucleolus is a protein-dense, membrane-less organelle, containing predominantly components of the rDNA transcription machinery, factors involved in nascent rRNA processing and ribosomal proteins (Montacie et al, 2017; Pendle et al, 2005). The tripartite ultrastructure of the nucleolus has been elucidated by electron microscopy (EM) using differential contrasting methods (de Carcer \& Medina, 1999; Medina et al, 2000). Individual conserved domains facilitating the spatial separation of rDNA processing comprise fibrillar centers (FC), dense fibrillar components (DFC) and granular components (GC) (Lafontaine, 1958; Perry, 1966). The transcription arises at the border of FC and DFC while nascent rRNA is processed in the DFC and GC (McKeown \& Shaw, 2009; Melcak et al, 1996; Smirnov et al, 2014). Two types of FCs were described so far in plant meristematic cells: heterogenous and homogenous (Risueno et al, 1982). Heterogenous FCs contain condensed as well as loosened rDNA chromatin, while homogenous FCs consist of decondensed rDNA available for transcription (McKeown \& Shaw, 2009), suggesting another level of compartmentalisation of nucleolar rDNA fraction. In mammals, nucleolar size correlates with the RNA pol I activity, determining the level of rDNA transcription and reflecting the cell differentiation state (Derenzini et al, 1988b; Watanabe-Susaki et al, 2014). Transcriptional activation of rDNA is maintained epigenetically. In $A$. thaliana, disruption of genes encoding HISTONE DEACETYLASE 6 (HDA6), histone methyltransferases TRITHORAX-RELATED PROTEIN 5 and 6 (ATXR5, ATXR6; H3K27me deposition) or SU(VAR)3-9 HOMOLOG 5 and 6 (SUV5, SUV6; H3K9me2 deposition) shows changes in rDNA histone methylation and acetylation and deregulation of rDNA transcriptional control (Earley et al, 2010; Mohannath et al, 2016; Pontvianne et al, 2012; Probst et al, 2004). At the global level, repressive marks H3K27me1 and 
$\mathrm{H} 3 \mathrm{~K} 9 \mathrm{me} 2$ are enriched in ribosomal genes of A. thaliana, reflecting the high proportion of inactive genes; the levels of H3K4me3 and H3K27me2-3 are detectable but much lower (Mathieu et al, 2005). Similarly, both H3K4me3 (active) and H3K9me2 (repressive) histone modifications regulate the rDNA epigenetic states in the hybrid of A. thaliana and A. arenosa called A. suecica (Lawrence et al, 2004).

Another layer of epigenetic regulation is the incorporation of $\mathrm{H} 3$ histone variants. Consistent with the transcriptional activity of nucleolar rDNA, transiently expressed AtH3.3-GFP formed nucleolar foci in tobacco leaves and similar sites were found in A. thaliana AtH3.3-FLAG-HA tagged lines (Nie et al, 2014; Shi et al, 2011). Even though H3.1 and H3.3 differ only in four amino acids (Ahmad \& Henikoff, 2002; Shi et al, 2011), these minor differences play essential role during their post-translational modification and functional specification. H3.1 is incorporated during the S phase by histone chaperone CHROMATIN ASSEMBLY FACTOR 1 (CAF-1) (Campos et al, 2015; Smith \& Stillman, 1989; Tagami et al, 2004) whereas H3.3 associates with actively transcribed genes and it is deposited independently of replication by other histone chaperones (Duc et al, 2017; Duc et al, 2015; Nie et al, 2014). Chromatin immunoprecipitation - sequencing (ChIP-seq) revealed that H3.1 is abundant in heterochromatin regions, transposable elements, sites containing repressive histone marks $\mathrm{H} 3 \mathrm{~K} 9 \mathrm{me} 2, \mathrm{H} 3 \mathrm{~K} 27 \mathrm{me} 1$ or $\mathrm{H} 3 \mathrm{~K} 27 \mathrm{me} 3$ and genes with methylated promoter regions (Stroud et al, 2012). H3.3 enrichment, in comparison, correlated with CG methylation in gene bodies, H3K4me1, ubiquitination of H2B and its levels peak at the 3' end of actively transcribed genes (Stroud et al, 2012). In addition, positioning of nucleosomes inside active rDNA repeats has been long discussed. Some studies suggest that this region is less associated with nucleosomes or even devoid of them compared to the inactive rDNA genes or intergenic spacers (IGS) (Zentner et al, 2011). Others demonstrated a nucleosome shift at the promoter site regulating rDNA transcription (Li et al, 2006; Zhao et al, 2016) and the presence of non-nucleosomal complexes, summarised in (Schofer \& Weipoltshammer, 2018).

Our knowledge of the structure of nucleolar rDNA, its spatial separation and chromatin organisation is very limited in plants. Due to inherent technical problems with ChIP-seq analysis of repetitive genomic regions, little is known about the distribution of epigenetic marks and histone variants in active rDNA as this approach only provides an average image of mixed active and inactive rDNA (Vaquero-Sedas et al, 2012). In this paper, we combine well-established biochemical techniques with innovative microscopy approaches to study the chromatin state of rDNA copies inside the nucleolus and on its periphery. In order to reduce the complexity of rDNA chromatin, particularly of the epigenetic states coexisting in one rDNA cluster, we employed experimental lines with reduced amount of rDNA copies containing 15-20\% of initial rDNA amount (Pavlistova et al, 2016). We show that NOR4-derived rDNA is organised in nucleolar foci in WT, formed as a consequence of rDNA compaction and associates with both H3.1 and H3.3 histone variants. Taking advantage of a low rDNA copy plant line, we show that both nucleolar foci and perinucleolar chromocenters are the result of the folding of rDNA in plant nucleoli. We combine ChIP-seq analysis of rDNA chromatin in seedlings with microscopic, single-locus detection of the rDNA epigenetic profiles on extended chromatin fibers and show that a portion of rDNA genes in a single NOR can contain various combinations of histone modifications. Moreover, analysis of ChIP-seq data indicates the presence of a wellpositioned nucleosome at the transcription start site and dynamic changes of $\mathrm{H} 3$ histone variants abundance at rDNA that might be key for its transcriptional regulation.

\section{Results}

\section{Both histone $\mathrm{H} 3$ variants are found in the nucleolus and colocalise with rDNA}

The nucleolar rDNA is largely decondensed but not structure-free. In proliferating root cells a network like structure containing decondensed part and condensed foci occurs, as revealed by Structured Illumination Microscopy - SIM (Dvorackova et al, 2018). We refer to these condensed foci inside of the nucleoli throughout the text as nucleolar foci (NF) while we call condensed foci on the nucleolar periphery perinucleolar chromocenters. The detailed chromatin organisation of this network, however, remains poorly understood. Although it was shown that HTR5-GFP (H3.3) forms nucleolar foci in differentiated cells, the situation in proliferating cells or the presence of HTR3-GFP (H3.1) in the nucleolus were not further explored (Nie et al, 2014; Shi et al, 2011). Therefore, we decided to investigate the contribution of individual H3 variants $(\mathrm{H} 3.3, \mathrm{H} 3.1)$ to the structure of $\mathrm{NF}$ and to the structure of perinucleolar chromocenters containing NOR4. We used two experimental plant lines - one expressing H3.1-GFP and H3.3-RFP (referred to as 
RedGreen) (Otero et al, 2016) and the second containing H3.1-RFP and H3.3-GFP (referred to as GreenRed). Imaging the root nuclei of Arabidopsis seedlings (up to 10-d-old) revealed that both histone variants are present in the NF (RedGreen, GreenRed; Fig. 1A, SI appendix, Fig. S1A) as well as in perinucleolar chromocenters (Fig. $1 A$ and $D$ ). Nucleolar signals of H3.1 and H3.3 were not identical in both tested lines. In GreenRed plants we detected the presence of smaller, monovariant foci labelled only by H3.3 (22 / 67 nuclei), while this pattern was rare in RedGreen plants (6 / 65 nuclei; Fig. $1 B$, SI appendix, Fig. S1A). This discrepancy might be caused by unbalanced levels of transgene expression, as shown previously by western blotting (Otero et al, 2016). We propose that the majority of cells contain $\mathrm{H} 3.3$ variant in all NF as well as in perinucleolar chromocenters. Most of these sites are also labelled by H3.1 variant and only a subset of cells shows small monovariant H3.3 labelled NF.

We further decided to investigate whether $\mathrm{H} 3.1$ and $\mathrm{H} 3.3$ are positioned in close proximity inside of the NF or form mutually exclusive environments and whether this positioning resembles the situation in the nucleoplasm. We employed Forster Resonance Energy Transfer approach combined with Fluorescence Lifetime IMaging measurements - FLIM-FRET - detecting protein-protein interactions within $10 \mathrm{~nm}$. We evaluated average fluorescence lifetimes $(\tau)$ in the nucleoplasm and in the NF, using GFP and mRFP as a donor-acceptor pair. In the control experiment (H3.1-GFP plants), GFP average lifetime was $\tau=2481 \pm 21.34$ ps $(n=41)$, while in the RedGreen line $(n=27)$ shortening of donor lifetime was detected (Fig. 1C). The maximum of GFP lifetime observed in RedGreen reached $\tau=2189 \mathrm{ps}$, corresponding to $12 \%$ and $36 \%$ of FRET, respectively. Since we noticed that nuclei differed in fluorophore levels, known to affect FRET (Gordon et al, 1998), we measured fluorescence intensity of H3.1-GFP and H3.3-RFP in the images acquired prior to FLIM-FRET measurement to calculate acceptor over donor (AOD) ratio. Values of this parameter varied from $20 \%$ to $86 \%$. More pronounced lifetime shortening correlated with the growth of AOD ratio (Fig. $1 C$ ), explained by larger probability of energy transfer between acceptor and donor fluorophores. We repeated the experiment on the line expressing H3.3-GFP as a donor and H3.1-mRFP as an acceptor (GreenRed line). Measured FRET value was only 4.3\% (SI appendix, Fig. S1B) as explained by the much higher expression of donor H3.3 than that of acceptor H3.1 in GreenRed line (Otero et al, 2016). In both GreenRed and RedGreen lines, the average lifetime of GFP-tagged histones in NF did not differ from the lifetime in nucleoplasm (SI appendix, Fig. S1C). This data suggests that these sites do not show any specific interaction or accumulation of H3.1 and H3.3 when compared to the nucleoplasm.

To test whether the NF correspond to the rDNA, we used immunodetection (IF) of individual H3 variants combined with fluorescence in situ hybridisation (FISH). Nucleoli-enriched samples isolated from H3.3-Myc and H3.1-Myc plant lines were imaged using STimulated Emission Depletion microscopy (STED). We observed that all H3.1 and H3.3-containing NF colocalised with condensed rDNA loci (Pearson correlation coefficient $\mathrm{r}=0.45 ; \mathrm{SD}=0.22$ and $\mathrm{r}=0.38 ; \mathrm{SD}=0.13$ for $\mathrm{H} 3.1$ and $\mathrm{H} 3.3$, respectively); (Fig. 1 $D-F)$. The values of Pearson correlation coefficient were expected to be relatively low due to differences in signal intensities (rDNA signal is much stronger than that of $\mathrm{H3}$ immunodetection) and enhanced resolution of STED microscopy.

\section{Decrease in rDNA amount leads to the loss of H3.3 labelled nucleolar foci in actively dividing root tissue}

To further investigate the character of NF we employed the low-copy rDNA line 9 (L9) generated in our previous work (Pavlistova et al, 2016) and introduced a H3.3-GFP construct into this line. L9 contains only about $\sim 15 \%$ of rDNA copies (compared to the wild-type - WT, SI appendix, Fig. S2) and the majority of these copies are considered active. We found that in the control WT line, H3.3-GFP forms NF, similarly to RedGreen plants (Fig. 2A(1); SI appendix, Fig. S3 $A$ and $C$ ). In the L9 these NF were less frequent, particularly in the meristematic root zone (Fig. 2A(2); SI appendix, Fig. S3B). When we counted the H3.3 signals in the nucleolus, we noticed a significant decrease in the number of $\mathrm{H} 3.3$ foci in the L9 (Fig. 2B). Most of the scored from the low rDNA copy lines were observed in the elongation zone and the root tip, where the cells undergo endoreduplication and differentiate (SI appendix, Fig. S3 $C$ and D). This data suggested that the formation of H3.3-labelled NF relates to the total rDNA amounts.

We next performed whole-mount in situ hybridisation on seedlings using a rDNA probe and assessed the distribution of ribosomal genes in root cells. In WT, we detected rDNA signals both inside of the nucleolus and on the nucleolar periphery. As expected, perinucleolar rDNA signals were universally much stronger than intranucleolar ones (Fig. 2C(1); SI appendix, Fig. S3F). In the low-copy line 6 (L6), where only 
50-70 copies of rDNA occur in 2C cells (Pavlistova et al, 2016), perinucleolar rDNA signals were mostly absent and decondensed nucleolar rDNA sites became more pronounced (Fig. 2C(2); SI appendix, Fig. S3H).

At this level of resolution, we could not investigate the nucleolar rDNA in more detail. Therefore, we used STED microscopy to gain a greater insight into the rDNA organization in WT and low-copy lines. The current idea of rDNA organization is based on the analysis of differentiated cells in WT plants (Pontvianne et al, 2013), where a large proportion of rDNA is inactive and which does not accurately capture the full spectrum of rDNA spatial arrangements. Improved resolution $(\sim 200 \mathrm{~nm})$ of SIM, was recently used to examine the details of rDNA architecture in replicating cells (2C - 4C)(Dvorackova et al, 2018). STED ( $50 \mathrm{~nm}$ resolution) revealed that most of the nucleoli contain NF brightly stained with DAPI and labelled by rDNA probe, resembling nuclear chromocenters. STED experiments clearly demonstrated the presence of smaller DAPI-free rDNA foci that can be found alongside larger rDNA/DAPI bright sites (Fig. $1 D$ ). When we examined isolated nucleoli, WT and low-copy plants displayed several patterns of rDNA organization, owing to the differentiation / endoreduplication status of nuclei from which the nucleoli were isolated (Fig. $2 D$ ): 1) nucleoli with a big number of small, diffuse nucleolar rDNA foci occurring selectively in the lowcopy line (Fig. 2 D1 and D2), 2) nucleoli showing bigger and smaller foci (Fig. 2 D3-D6), 3) nucleoli with large perinucleolar chromocenters and compact nucleolar foci without dispersed smaller signals (Fig. 2 D7$D 9)$. The latter two types of organization occur both in low-copy and WT plants. In these samples, the larger rDNA foci were DAPI labelled (Fig. 2E). As some nucleoli isolated from low-copy rDNA plants show similar pattern of rDNA organisation as WT (type 2 and 3), we assume that nucleolar morphology reflects the ratio of actively transcribed to total rDNA repeats. This suggests that the differences in the organization of rDNA between WT and low-copy lines are most pronounced in meristem nuclei that have the highest ratio of active rDNA copies to total rDNA content (Dvorackova et al, 2018). Consistently, the loss of the H3.3 NF occurs predominantly in the meristem root zone (SI appendix, Fig. S3 $A$ and $B$ ). Once the ratio of active/inactive rDNA is low enough, the morphology of WT and low-copy nucleoli is alike (SI appendix, Fig. S3 E-H).

\section{H3.3 marks the nucleosome at rDNA transcription start site in actively dividing tissue}

Since epigenetic mechanisms are essential in establishing active and inactive states of rDNA copies, we decided to investigate the distribution of histone variants and histone modifications along the rDNA repeats. We took advantage of the publicly available ChIP-seq data sets collected from young and mature leaves (actively dividing tissue with higher rDNA transcription and non-dividing tissue with lower rDNA activity, respectively) (Wollmann et al, 2017). The data were processed with an algorithm optimised for the analysis of rDNA (Zentner et al, 2011) and regions where irrelevant reads could map to (called low mappability regions, LMR, SI appendix Fig. S3A) were excluded from our analysis (described in SI appendix, Methods). We found that in dividing tissue, the distribution pattern as well as total levels of H3.1 and H3.3 signal were similar along the rDNA coding sequence (Fig. $3 B$ and $C$ ). In the non-dividing tissue, the total H3.1 level was enriched over H3.3 suggesting the silencing of a larger subset of rDNA copies typical during transcriptional downregulation. A characteristic feature of actively dividing tissues was a H3.3 peak of approx. single nucleosome width at the transcription start site, position $(-73,167)$ (Fig. 3C). This peak was lost in the nondividing tissue, since the levels of H3.3 at the TSS dropped below 0 (Fig. 3C). We did not observe the accumulation of H3.3 towards the 3'end of the gene, commonly observed in RNA pol II transcribed genes (Fig. 3D).

\section{Wild-type and low-copy rDNA lines show similar distribution of $\mathrm{H} 3$ in the $\mathrm{rDNA}$ repeat}

Since the publicly available ChIP-seq data are performed on plant lines containing WT rDNA amounts with the majority of inactive genes, we performed a ChIP-seq experiment on the low-copy line L9 (Pavlistova et al, 2016). This experiment revealed that the H3 occupancy pattern along the rDNA sequence is identical in L9 and WT (Fig. 3E): histone H3 is the lowest at the promoter region at the position (-323, 123) relatively to TSS followed by an abrupt signal increase at the rest of the mappable promoter region (73, 439; Fig. $3 F)$. The two peaks with the highest signal were detected at the beginning $(4342,5317)$ and in the middle $(5317,6427)$ of $25 \mathrm{~S}$ rDNA sequence. Another feature of this profile is the increased signal level at the end of the coding sequence $(7217,7677$; Fig. $3 G)$. 


\section{H3K4me3 and H3K27me3 levels are low in rDNA and mark promoter as well as the gene body}

We next focused on the distribution of different histone modifications by a) using available public ChIP-seq datasets on WT and by b) employing the ChIP - Dot blot approach on the WT and two low-copy lines (L6, L9). We started with the dataset from young rosette leaves (PRJNA218138; (Chica et al, 2013)) to determine the distribution of H3K4me3 and H3K27me3 histone marks. The levels of both marks were very low in the rDNA locus, as confirmed by both Dot blot (SI appendix, Fig. S5 $A$ and $B$ ) and ChIP-seq, when compared to other genomic regions (SI appendix, Fig. S6). The distribution pattern was similar for both modifications (Fig. $4 A$ and $B$ ). The lowest signal was detected at the promoter region of a single nucleosome size at $(-323$, -123) with respect to TSS, followed by abrupt signal increase at the remaining mappable part of the promoter adjacent to TSS $(-73,439)$ similarly to reported total H3 pattern. Again, largest signals were in the beginning and in the middle of $25 \mathrm{~S}$ rDNA coding sequence at same positions as total $\mathrm{H} 3$ enrichment $(4342,5317 ; 5317$, 6427 ) and a large peak at the end of the coding sequence, partially overlapping the $\mathrm{H} 3$ peak $(7347,7677)$. While both marks are usually associated with regulation of the promoter activity, we did not observe a significant increase in the promoter sequence in comparison with the coding sequence. This data shows very low levels of H3K4me3 and H3K27me3 in both WT and low-copy plants, suggesting a minor role in the regulation of rDNA activity.

\section{H3K9me2 and H2A.W are abundant in the rDNA coding sequence while H3K9me2 is lower at the promoter}

The distribution of heterochromatin histone mark $\mathrm{H} 3 \mathrm{~K} 9 \mathrm{me} 2$ and histone variant $\mathrm{H} 2 \mathrm{~A} . \mathrm{W}$ was assessed using publicly available datasets (PRJNA162669; (Moissiard et al, 2012) and PRJNA219442;(Yelagandula et al, 2014), respectively). Both marks are strongly enriched in the whole rDNA locus (Fig. $4 C$ and $D$ ), with levels comparable to the regions of their highest enrichment such as centromeres and transposable elements $(S I$ appendix, Fig. S6). Their signal is equally distributed in the rDNA coding sequence without any well-defined peaks. H3K9me2 levels are lowered by half at the promoter in comparison with the coding sequence, reaching the lowest values before the TSS $(-273,-123)$. There is a decrease in H2A.W abundance before the TSS but it is not as pronounced and visible as with previously described modifications. Dot-blot analysis revealed a sharp decrease of H3K9me2 levels in 18S region of low-copy lines L6 and L9 when compared to WT (Fig. $4 E-G)$. There is less $\mathrm{H} 3 \mathrm{~K} 9 \mathrm{me} 2$ in the transcription start site than in the coding sequence, corroborating results obtained from ChIP-seq data.

\section{Epigenetic profile of the rDNA cluster}

We next analysed whether NF accumulate any specific histone modifications using immunofluorescence (IF) detection. We found that large NF were predominantly marked by H3K9me2 (Fig. $5 A 1$ and $A 2$ ), similarly to the nuclear chromocenters while some of the smaller NF did not show H3K9me2 accumulation (Fig. $5 A 3$ and $A 4$ ). In WT, H3K9me2 labelling always occurred in perinucleolar chromocenters and in approximately $50 \%$ of NF (22/44; Fig. $5 B$ ). Low-copy line L6 showed smaller proportion of NF labelled by H3K9me2 (8 / 32; Fig. 5B). We further investigated whether the H3K27me3 and H3K4me3 also occur in the nucleoli. Regardless of a large number of studies containing some H3K27me3 and H3K4me3 IF data on plant nuclei (Lawrence et al, 2004; Mathieu et al, 2005), the presence of these two histone marks has neither been clearly shown nor excluded. In isolated nuclei, both H3K4me3 and H3K27me3 marks display typical diffuse distribution in the nucleoplasm (SI appendix, Fig. S7 $A$ and $B$ ). We reasoned that even if these marks contribute to rDNA transcriptional regulation, they represent a relatively small proportion of total rDNA repeats. With dSTORM microscopy we found that some nucleoli appear mostly devoid of these modifications while others show modest localisation of $\mathrm{H} 3 \mathrm{~K} 4 \mathrm{me} 3$ and $\mathrm{H} 3 \mathrm{~K} 27 \mathrm{me} 3$ inside nucleoli (Fig. $5 \mathrm{C}$ $F$ ). In this experiment, we used HILO illumination and stringent settings for localisation detection, excluding localisations with low localisation precision or low emission strength to minimize the detection of the signals coming from out-of-focus fluorophore blinking.

In order to explore mutual positions of histone modifications along the rDNA repeat, we optimised the method of extended chromatin fibers and investigated selected histone modifications in WT plants. First, we validated FISH detection of rDNA in chromatin fiber preparations (SI appendix, Fig. S7C). Using 
combined FISH/IF on chromatin fibers, we detected rDNA clusters entirely devoid of the studied histone marks (SI appendix, Fig. S8) or observed rDNA clusters with varying arrangement of repressive H3K27me3 and active H3K4me3 marks (Fig. 5G). As these marks sometimes colocalized along one fiber they are likely to correspond to the bivalent chromatin regions. Repressive mark H3K9me2, in contrast, did not colocalize with H3K4me3, as expected (Fig. 5H). Surprisingly, we did not notice clear boundaries separating the repressive and active chromatin states (Fig. $5 H(7)$ ).

\section{Discussion}

\section{Perinucleolar chromocenters and nucleolar foci}

Chromocenters are nuclear domains where silent, condensed and heavily methylated heterochromatic parts of the genome accumulate (Fransz et al, 2006; Soppe et al, 2002 ). Nearly 50\% of ribosomal genes are typically silenced and reside in these chromocenters (Fransz et al, 2002; Fransz et al, 2006; Pavlistova et al, 2016; Pontvianne et al, 2013; Soppe et al, 2002; Tessadori et al, 2007). In the Col-0 Arabidopsis ecotype, inactive NOR2 assembles into chromocenters at the nuclear periphery (Dvorackova et al, 2018; Pavlistova et al, 2016) while the active NOR4 forms chromocenters at the periphery of the nucleolus (Fransz et al, 2002; Pontvianne et al, 2013). Active rDNA genes loop into the nucleolus from perinucleolar chromocenters in a decondensed form (Highett et al, 1993; Leitch et al, 1992), reminiscent of euchromatin loops observed in the nucleoplasm (Fransz et al, 2002). Therefore, the presence of both H3.1 and H3.3 histone variants in the perinucleolar rDNA-containing chromocenters possibly reflects the complexity of rDNA organisation at NOR4, where both active and inactive copies coexist. We focused our attention on the fact that chromocenter-like structures, here called nucleolar foci (NF), occur inside the nucleolus. These foci differ in size and chromatin structure and correspond to rDNA (Fig. 1D and 2D). Although the presence of NF is evident from many cytological studies and the idea of active nucleolar rDNA being interconnected with condensed sites was previously introduced (Highett et al, 1993; Neves et al, 2005), it has not been much explored. We show that NF occur more often in the nuclei with a higher ploidy level, while nuclei of the dividing zone show nucleolar rDNA signals lacking apparent condensed sites (Fig. 2C). Interestingly, we found that the epigenetic profile of all NF is not identical. Larger foci are brightly stained with DAPI and either contain both $\mathrm{H} 3$ variants and $\mathrm{H} 3 \mathrm{~K} 9 \mathrm{me} 2$ or they lack $\mathrm{H} 3 \mathrm{~K} 9 \mathrm{me} 2$ (Fig. $5 A$ ). In some cells, we identified a subpopulation of NF that seemed to contain only H3.3. The co-occurrence of H3.1 histone variant and $\mathrm{H} 3 \mathrm{~K} 9 \mathrm{me} 2$ is indicative of silent chromatin while $\mathrm{H} 3.3$ was shown to coexist with euchromatin marks, such as H3K4me3, H3K36me3 and histone acetylation (Roudier et al, 2011; Sequeira-Mendes et al, 2014). In agreement with the general idea of $\mathrm{H} 3 \mathrm{~K} 9 \mathrm{me} 2$ distribution inside the inactive rDNA, summarised in (Neves et al, 2005), fewer H3K9me2 labelled NF occurred in lines with reduced rDNA copy number, where the overall abundance of $\mathrm{H} 3 \mathrm{~K} 9 \mathrm{me} 2$ was also lower (Fig. $4 E$ and $F ; 5 A$ and $B$ ). This supports the model that inactive rDNA copies assemble into a heterochromatic state not only at the perinucleolar chromocenters but also inside the nucleolus (at NF) and that their epigenetic profile is similar. Nevertheless, NF lacking the $\mathrm{H} 3.1$ variant and $\mathrm{H} 3 \mathrm{~K} 9 \mathrm{me} 2$, represent another type of chromatin structure. When viewed in isolation, the occurrence of $\mathrm{H} 3.3$ in NF could be interpreted as a sign of active transcription, facilitating the access of RNA pol I to the promoter and allowing flexible epigenetic regulation. We suppose that $\mathrm{H} 3.3$ presence in rDNA chromocenters is necessary for its rapid regulation, akin to environmental response genes, as shown previously (Liu et al, 2014; Wollmann et al, 2017).

Whether the condensed H3.1 and H3.3 foci inside the nucleolus truly label transcriptionally active rDNA or relate to the folding of rDNA copies is unclear. In differentiated cells, occurrence of H3.3 labelled NF correlated with rDNA transcription, as concluded from actinomycin-D (ActD) treatment leading to the depletion of H3.3 from the nucleolus (Shi et al, 2011). However, ActD was shown to induce nucleolar stress response (van Sluis \& McStay, 2019), during which intranucleolar rDNA migrates to the periphery of the nucleolus (Franek et al, 2016; Harding et al, 2015). Therefore, the removal of H3.3 from nucleoli after ActD does not necessarily relate to the inhibition of RNA pol I. We analysed the root meristem, where most cells are diploid and the rDNA copy number is not amplified by polyploidization. H3.3 chromocenters were present in the plants containing WT rDNA copy number and absent in low rDNA copy lines, where the majority of rDNA copies is active (Fig. 2A). Meristem nuclei of low rDNA copy lines contained only the nucleolar and decondensed rDNA fraction suggesting that the formation of $\mathrm{H} 3.3$ chromocenters might be 
connected to the folding of non-transcribed rDNA and possibly correspond to the semi-condensed rDNA state, as suggested previously (Highett et al, 1993). Folding of rDNA as a mechanism of rDNA dosage control is a generally accepted concept, providing another layer of epigenetic regulation (Highett et al, 1993; Chen et al, 1998). The complexity of nucleolar rDNA, however, often impedes the visualization of the active rDNA due to the signals from condensed repeats. STORM, as we show, could bring a new insight into the rDNA folding process as it enabled detection of highly decondensed genes even in the nuclei where the nucleolus is small and rDNA condensed sites dominate (SI appendix, Fig. S9).

The super-resolution STED microscopy is another suitable and less demanding approach to capture the heterogeneity of the types of rDNA organization and different degrees of rDNA condensation. It revealed the presence of smaller rDNA signals dispersed in the nucleolus (Fig. 2D) together with NF. We suggest that the ratio of active to inactive ribosomal copies is the primary determinant of the rDNA organization inside of nucleoli. Once the requirements for rDNA transcription decrease, the folding of unnecessary repeats into NF or perinucleolar rDNA chromocenters is initiated. This explains why both types of structures occur also in the low-copy line, particularly in the endocycling root cells (SI appendix, Fig. S2 D). Terminal stage of the "packing" process is represented by the formation of a few large perinucleolar chromocenters with or without NF (Fig. 2 D7 and D9). The ratio of the nucleolar volume to the remaining nucleoplasm itself represents an indicator of the transcriptional activity of the nucleolus (Derenzini et al, 1988a; Watanabe-Susaki et al, 2014). We observed a connection between the size of nucleoli and rDNA distribution. This mostly manifests in large nucleoli, where small dispersed rDNA foci are common, and small nucleoli, where large rDNA foci are formed on the periphery of the nucleolus. Nevertheless, the regulation of rDNA folding remains elusive. Why rDNA sometimes assembles into NF while at other times it forms perinucleolar chromocenters is an open question and it is currently unknown what molecular players are mediating this process.

We reasoned that several chromatin subtypes might exist in different NF. Besides H3.3, we did not identify any active epigenetic marks in NF that would support the existence of chromocenters with uniquely active chromatin characteristics. The H3K4me3 signal was low and dispersed, with no signs of localised foci. $\mathrm{H} 3 \mathrm{~K} 27 \mathrm{me} 3$, that could potentially indicate the flexible regulation of a subset of nucleolar rDNA, was also low and not associated with chromocenters (Fig. 5).

\section{The role of histone modifications in rDNA organization and transcription}

In Arabidopsis, nine chromatin states, defined by different levels of DNA methylation, histone posttranslational modifications and histone variants were described by genome wide analyses (Roudier et al, 2011; Sequeira-Mendes et al, 2014). Given the complexity of rDNA organisation, identification of individual chromatin states inside its large repetitive clusters is challenging. The same way the clusters of inactive rDNA copies on the nucleolar periphery obscure the microscopic analysis of the active rDNA fraction, active and poised rDNA fractions are masked by inactive rDNA in ChIP-seq analysis. IF data and ChIP-PCR show that two repressive marks H3K9me2, H3K27me1 are the most abundant modifications in nuclear chromocenters, including perinucleolar rDNA sites (Earley et al, 2010; Jacob \& Michaels, 2009). The ChIP-seq analysis revealed that besides $\mathrm{H} 3 \mathrm{~K} 9 \mathrm{me} 2, \mathrm{H} 2 \mathrm{~A}$.W is also strongly enriched all over the rDNA repeat (Fig. $3 C$ and $D$ ). The broad distribution pattern of $\mathrm{H} 2 \mathrm{~A}$.W and $\mathrm{H} 3 \mathrm{~K} 9 \mathrm{me} 2$ seems characteristic for plants as in human and mice rDNA, repressive modifications form separate peaks (Zentner \& Henikoff, 2014). Arabidopsis H2A.W is known to promote higher order chromatin condensation and it can be deposited independently of H3K9me2 (Yelagandula et al, 2014). Therefore, there might be several epigenetic mechanisms regulating the activity of rDNA. The exact nature of the molecular switch between active and silenced states in rDNA is unknown, but it does involve active and repressive histone marks (Lawrence et al, 2004; Pontes et al, 2007; Probst et al, 2004). Activation of silenced rDNA copies is known to be mediated by histone acetylation and DNA demethylation (Earley et al, 2010; Chen \& Pikaard, 1997). One layer of specific rDNA regulation is ensured by the nucleolar localisation of histone modifiers, including histone deacetylase HDA6 or the histone methyltransferase SuvR4 (Thorstensen et al, 2006) (Pontvianne et al, 2013). The latter introduces H3K9me2 and its presence in the nucleolus could relate to the occurrence of H3K9me2 labelled NF (Fig. 5 A). Why some histone modifiers and histone marks are found in the nucleolus while others are not, remains unclear and the complete view of rDNA regulation is still missing. For example, nucleolar localisation was shown for H3S10 phosphorylation in tobacco cells (Granot et al, 2009), though it is known to associate with the mitotic progression (Demidov et al, 2009). On the contrary, two euchromatic marks H3K4me3 and 
H3K27me3 (active and repressive, respectively) were not clearly visible in the nucleolus (Mathieu et al, 2003; Wollmann et al, 2017). In dSTORM experiments, we could detect intranucleolar localisation of these marks (Fig. $5 C-F$ ) and low signal emerged also in the ChIP-seq data (Fig. $4 A$ and $B$ ). IF-FISH on chromatin fibers, in comparison, showed quite homogeneous distribution of H3K4me3 along the rDNA clusters (Fig. 5 $G$ and $H$ ). The use of extended chromatin fibers, as we show, helps explore the potentially hidden rDNA epigenetic patterns and provides high resolution image of the mutual distribution of individual histone modifications. We also detected $\mathrm{H} 3 \mathrm{~K} 9 \mathrm{me} 2$ foci well separated from H3K4me3 sites and colocalisation between H3K4me3 and H3K27me3 (Fig. $5 G$ and $H$ ). The initial model of chromatin organisation in Arabidopsis considered H3K4me3 and H3K27me3 as mutually exclusive (Roudier et al, 2011; Schwartz \& Pirrotta, 2007), but more recent studies revealed that these two marks can coexist and occur in stress response genes (Liu et al, 2014; Sequeira-Mendes et al, 2014). This corresponds with a view that bivalent (poised) chromatin allows for a more dynamic transcriptional regulation, both in the case of differentiation (Bernstein et al, 2006) and environmental response (60). It is therefore evident that several different epigenetic states exist in rDNA, shown here and in (Earley et al, 2010; Pontvianne et al, 2010; Pontvianne et al, 2013; Probst et al, 2004)

Apart from the regulation mediated via post-translational modification, histone variants contribute to the regulation of rDNA transcription. In RNA pol II transcribed genes, H3.3 is known to associate with active genes, affect gene body methylation and to be enriched in transcription end site (Ahmad \& Henikoff, 2002; Stroud et al, 2012; Wollmann et al, 2017). H3.3 distribution pattern in rDNA differs from RNA pol II transcribed sites: it shows H3.3 enrichment in the TSS and lack of increase towards the 3' end (Fig. $3 C$ and D). We suggest that H3.3 in the TSS could be characteristic for RNA pol I transcribed genes and that rDNA promoter is an important regulatory element. In addition to $\mathrm{H} 3.3$, both $\mathrm{H} 3 \mathrm{~K} 4 \mathrm{me} 3$ and $\mathrm{H} 3 \mathrm{~K} 27$ me 3 displayed a peak at the TSS while the levels of $\mathrm{H} 3 \mathrm{~K} 9 \mathrm{me} 2$ dropped in this region. Another Pol 1 feature was a strong depletion of all studied histone marks before the TSS. This is reminiscent of the mouse and human rDNA, regulated via upstream control element (UCE), where a narrow peak of activating modifications was reported (Zentner \& Henikoff, 2014; Zentner et al, 2011).

Assigning rDNA to any known chromatin state is not straightforward. A large subset of rDNA repeats seems reminiscent of chromatin state 9, characterised by high levels of H3K9me2, H3K27me1, CG methylation and H3.1 histone variant (Sequeira-Mendes et al, 2014). Smaller subset of rDNA is similar to the chromatin state 2, due to the co-occurrence of H3K4me3 and H3K27me3 (Sequeira-Mendes et al, 2014). The distribution of H3K4me3 and H3K27me3 fits into the model where chromatin states form only short, several kb long segments interspersed with each other (Roudier et al, 2011) and it might represent a poised rDNA state. State 2 is typical for promoters and intergenic regions (Sequeira-Mendes et al, 2014), but whether this is the case for rDNA is currently unclear. Moreover, our data indicate features that make rDNA chromatin signature unique, such as $\mathrm{H} 3.3$ abundance in rDNA perinuclear chromocenters and nuclear foci or $\mathrm{H} 3.3$ specific peak at the TSS. Interestingly, we observe presence of H3K9me2 in nucleolar foci that are universally labelled with H3.3, suggesting the existence of another chromatin environment. It is therefore evident that rDNA exists in a variety of chromatin states.

Overall, this paper uses new methodical approaches to tackle long-stranding questions in the organization and epigenetic regulation of ribosomal DNA. We show that both H3.1 and H3.3 histone variants occupy the majority of nucleolar foci and perinucleolar chromocenters, some of these sites are clearly repressive in nature since they display $\mathrm{H} 3 \mathrm{~K} 9 \mathrm{me} 2$ accumulation. Importantly, we employed low-copy rDNA lines to demonstrate that nucleolar foci are formed by the compaction of rDNA in nucleoli, a process initiated once the ratio of active and inactive copies reaches a certain threshold. We captured the heterogeneity of rDNA arrangements and folding in various cell types using super-resolution microscopy and show that intermediate levels of rDNA compaction occur, besides the often-referenced clustering of rDNA signals on the nucleolar periphery. Finally, we corroborate data from previous research showing the role of active (H3K4me3) and repressive (H3K27me3; H3K9me2; H2A.W) histone marks and variants in the control of rDNA activity. We suggest that integrating various methodical avenues in the study of rDNA physiology is required given the technical hurdles of individual methods and the complexity of rDNA regulatory processes which tie together spatial organization with changes in histone signature and transcriptional activity. In conclusion, this paper presents a complex investigation of rDNA physiology, from its organization in different cell types to the epigenetic variability of rDNA clusters. Key remaining open questions are a) which histone writers and histone readers are directly responsible for toggling on and off active and inactive states in rDNA b) why is 
rDNA preferentially folded into perinucleolar chromocenters in some cases and into nucleolar foci in others c) why is H3.3 histone recruited into rDNA condensed foci and whether its accumulation is the result of transcription or related to a different physiological process.

\section{Methods}

\section{Isolation of nuclei and nucleoli; chromatin fiber preparation}

Isolation of nuclei was performed as described in (Jackson et al, 1998). Isolated nuclei were centrifuged at $5000 \mathrm{~g}$ for $10 \mathrm{~min}$ at $4^{\circ} \mathrm{C}$ and resuspended in $75 \mathrm{mM} \mathrm{KCl}$. Chromatin fibers were extended as described in (Cohen et al, 2009), with minor modifications. Full protocol can be found in SI appendix, Methods. Nucleoli from 10-d-old seedlings were isolated based on the published protocol (Liang et al, 2012) with some modifications. Seedlings were fixed in 1\% paraformaldehyde (PFA) for $10 \mathrm{~min}$, quenched with $0.125 \mathrm{M}$ glycine and disrupted in Galbraight's buffer using ICA T25 Ultra Turrax homogenizer (11000 rpm, $90 \mathrm{~s})$. After filtration through $40 \mu \mathrm{m}$ cell strainers (Falcon, \#352340), nuclei were sonicated (Diagenode Bioruptor; 5 x 5 min cycles of $30 \mathrm{~s} \mathrm{ON} / 30 \mathrm{~s} \mathrm{OFF}$ ) and nucleoli were isolated by centrifugation through sucrose cushions as described.

\section{Immunofuorescence (IF) and FISH on nuclei and nucleoli, whole-mount FISH}

Immunofluorescence detection and FISH procedures on isolated nuclei and nucleoli is described in $S I$ appendix, Methods. For whole-mount FISH,10-d-old seedlings were fixed for 30 min in 1\% formaldehyde, 10\% DMSO, 0.5 mM EGTA in 1x PBS. Seedlings were rinsed twice in ethanol and 100\% methanol, then left in methanol O/N. Seedlings were washed 2 x 5 min in $1 \times$ PBS/0.1\% Tween-20 and $3 \times 5$ min in $2 x$ SSC. Seedlings were incubated $30 \mathrm{~min}$ in the mix of 2x SSC and HB50, 1:1 (HB= hybridization buffer, 10\% dextran sulfate, $50 \%$ deionized formamide in $2 \mathrm{x} \mathrm{SSC}) ; 30 \mathrm{~min}$ in $\mathrm{HB} 50$ and $1-2$ hours $\left(37^{\circ} \mathrm{C}, 300 \mathrm{rpm}\right)$ in $100 \mu \mathrm{L}$ of the probe mix ( $5 \mu \mathrm{L}$ of labelled rDNA probe in HB50). Seedlings were denatured for 4 min at $90^{\circ} \mathrm{C}$, cooled on ice for $3 \mathrm{~min}$ and hybridized $\mathrm{O} / \mathrm{N}$ at $37^{\circ} \mathrm{C}$. All subsequent washes were performed in the thermomixer (300 rpm): 2 x 5 min in 50\% formamide / 2x SSC at $42^{\circ} \mathrm{C} ; 1$ x 5 min in $2 \times$ SSC and 1 x 5 min in $1 \mathrm{x}$ PBS, both at $42^{\circ} \mathrm{C}$. Seedlings were then put onto a microscopic slide and covered with DAPI, $2 \mu \mathrm{g} / \mathrm{mL}$ in Vectashield.

\section{Chromatin immunoprecipitation (IP) and high throughput sequencing}

Nuclei isolation was performed using 2 g of 10-d-old seedlings. Nuclei were sonicated, 4 x 5 min cycle (30 $\mathrm{s}$ on/ 30 s off; Diagenode Bioruptor) with 4 min incubation on ice between sonication cycles. Samples were then centrifuged $4 \mathrm{x}$ at $20000 \mathrm{~g}$ for $10 \mathrm{~min}$ at $4^{\circ} \mathrm{C}$. Chromatin was pre-cleared by $\mathrm{O} / \mathrm{N}$ incubation with $40 \mu \mathrm{L}$ of Protein A/G beads (sc-2003, Santa Cruz biotechnology) on a stirring wheel at $4^{\circ} \mathrm{C}$. We prepared beadantibody complexes by $4 \mathrm{~h}$ incubation of protein $\mathrm{A} / \mathrm{G}$ agarose beads with $2 \mu \mathrm{g}$ of antibody (anti-histone H3 ab1791; anti-histone $\mathrm{H} 3 \mathrm{~K} 4 \mathrm{me} 3 \mathrm{ab8580}$; both Abcam) in total volume of $500 \mu \mathrm{L}$ of dilution buffer followed by $\mathrm{O} / \mathrm{N}$ blocking of antibody-bead complexes. DNA obtained after the protocol was processed by QIAquick PCR purification kit (Qiagen).

\section{Dot-blot, hybridization and nick-translation labelling of rDNA}

Dot-blot was performed according to previously described protocol (Mozgova et al, 2010). Primers and data evaluation are described in SI appendix, Methods. Probe labelling was adapted from (Mandakova \& Lysak, 2008) using $1 \mu \mathrm{g}$ of BAC as a template, isolated using Macherey Nagel NucleoBond® Xtra Midi. For visualization of the 45S rDNA loci the BAC clone T15P10 (GenBank AL095897/8) was used.

\section{FLIM-FRET}

10-d-old seedlings were transferred from growth plates onto microscopic slides and imaged using Zeiss LSM 780 (Carl Zeiss MicroImaging GmbH) microscope equipped with "In Tune" laser (488 nm for GFP, $561 \mathrm{~nm}$ for mRFP). FLIM was performed at $488 \mathrm{~nm}$ and $40 \mathrm{MHz}$ frequency and recorded using HPM 100-40 
(Becker\&Hickl, Berlin, Germany) hybrid detector, SPC-150 TCSPC (Becker\&Hickl) module and SPCM64 software. Lifetime images were further processed and analyzed in SPCImage 6.4 software (Becker\&Hickl).

\section{Microscopy and image analysis}

Whole-mount FISH and chromatin fiber images were acquired on an epifluorescence microscope Zeiss AxioImager Z2, using appropriate filters. Structured illumination microscopy was performed on Nikon 3D N-SIM microscope (inverted Nikon Eclipse Ti-E, Nikon) equipped with a Nikon CFI SR Apo TIRF objective (100x oil, NA 1.49). Two-color STED microscopy was performed on the Abberior STED system joined to a Nikon Eclipse Ti-E inverted confocal microscope. For dSTORM, samples labelled with either AF647 or CF680 (1:100 secondary antibody dilution) were imaged in the imaging buffer (50 mM Tris-HCl, $10 \mathrm{mM}$ $\mathrm{NaCl}, 10 \%$ (w/v) glucose, pH 8 with $50 \mathrm{mM} \beta$-mercaptoethylamine (MEA, 30070, Sigma-Aldrich), 1.1 $\mathrm{mg} / \mathrm{ml}$ Glucose Oxidase (G2133, Sigma-Aldrich) and $100 \mu \mathrm{g} / \mathrm{ml}$ Catalase (C40, Sigma-Aldrich).

Detailed description of plant material, growth conditions and all methods is presented in SI appendix, Methods.

\section{Acknowledgements}

We are grateful to: C. Gutierrez and S. Otero CBMSO, Madrid, Spain, for providing HTR5-mRFP/HTR3GFP, HTR4-myc and HTR3-myc plant lines; B. Desvoyes CBMSO, Madrid, Spain, for critical reading of the manuscript and fruitful discussions; F. Pontvianne, CNRS, Perpignan, France, for providing the FIB2YFP plant line; A. Probst, GReD, Clermont-Ferrand for the help with ChIP experiment optimization; D. Pánek, Imaging Methods Core Facility, for the support with the realization of spectral de-mixing experiment and analysis. We would like to acknowledge these core facilities (CF) for their support with obtaining data presented in this paper: CELLIM of CEITEC MU and Imaging Methods CF at BIOCEV, both supported by MEYS CR (LM2018129 Czech-BioImaging); Plants Sciences and Genomics of CEITEC MU.

MD and MF were supported by the Czech Science Foundation project 19-11880Y, by Ministry of Education, Youth and Sports of the Czech Republic - project INTER-COST (LTC18048) and by the European Regional Development Fund - Project "SINGING PLANT" (CZ.02.1.01/0.0/0.0/16_026/0008446). MO acknowledges financial support from the ERDF (project No. CZ.02.1.01/0.0/0.0/16_013/0001775).

\section{Author Contributions}

$\mathrm{KK}, \mathrm{MF}$ and MD performed the experiments and wrote the manuscript. MO assisted with super-resolution microscopy and subsequent image analysis. KD and JK assisted with ChIP-seq data analysis.

\section{Conflict of interest}

The authors declare no conflict of interest.

\section{References}

Ahmad K, Henikoff S (2002) The histone variant H3.3 marks active chromatin by replication-independent nucleosome assembly. Molecular cell 9: 1191-1200

Bernstein BE, Mikkelsen TS, Xie XH, Kamal M, Huebert DJ, Cuff J, Fry B, Meissner A, Wernig M, Plath K, Jaenisch R, Wagschal A, Feil R, Schreiber SL, Lander ES (2006) A bivalent chromatin structure marks key developmental genes in embryonic stem cells. Cell 125: $315-326$

Berr A, Schubert I (2007) Interphase chromosome arrangement in Arabidopsis thaliana is similar in differentiated and meristematic tissues and shows a transient mirror symmetry after nuclear division. Genetics 176: 853-863 
Borysko E, Bang FB (1951) Structure of the nucleolus as revealed by the electron microscope; a preliminary report. Bulletin of the Johns Hopkins Hospital 89: 468-473

Campos EI, Smits AH, Kang YH, Landry S, Escobar TM, Nayak S, Ueberheide BM, Durocher D, Vermeulen M, Hurwitz J, Reinberg D (2015) Analysis of the Histone H3.1 Interactome: A Suitable Chaperone for the Right Event. Molecular cell 60: 697-709

Cohen SM, Chastain PD, 2nd, Cordeiro-Stone M, Kaufman DG (2009) DNA replication and the GINS complex: localization on extended chromatin fibers. Epigenetics \& chromatin 2: 6

Copenhaver GP, Doelling JH, Gens S, Pikaard CS (1995) Use of RFLPs larger than $100 \mathrm{kbp}$ to map the position and internal organization of the nucleolus organizer region on chromosome 2 in Arabidopsis thaliana. The Plant journal : for cell and molecular biology 7: 273-286

de Carcer G, Medina FJ (1999) Simultaneous localization of transcription and early processing markers allows dissection of functional domains in the plant cell nucleolus. Journal of structural biology 128: 139151

Demidov D, Hesse S, Tewes A, Rutten T, Fuchs J, Ashtiyani RK, Lein S, Fischer A, Reuter G, Houben A (2009) Aurora1 phosphorylation activity on histone H3 and its cross-talk with other post-translational histone modifications in Arabidopsis. The Plant journal : for cell and molecular biology 59: 221-230

Derenzini M, Romagnoli T, Ceccarelli C, Eusebi V (1988a) Fixatives and silver stainability of NOR proteins at the light microscopic level. The journal of histochemistry and cytochemistry : official journal of the Histochemistry Society 36: 1453-1454

Derenzini M, Romagnoli T, Mingazzini P, Marinozzi V (1988b) Interphasic nucleolar organizer region distribution as a diagnostic parameter to differentiate benign from malignant epithelial tumors of human intestine. Virchows Archiv B, Cell pathology including molecular pathology 54: 334-340

Duc C, Benoit M, Detourne G, Simon L, Poulet A, Jung M, Veluchamy A, Latrasse D, Le Goff S, Cotterell S, Tatout C, Benhamed M, Probst AV (2017) Arabidopsis ATRX Modulates H3.3 Occupancy and FineTunes Gene Expression. Plant Cell 29: 1773-1793

Duc C, Benoit M, Le Goff S, Simon L, Poulet A, Cotterell S, Tatout C, Probst AV (2015) The histone chaperone complex HIR maintains nucleosome occupancy and counterbalances impaired histone deposition in CAF-1 complex mutants. Plant $J$ 81: 707-722

Dvorackova M, Raposo B, Matula P, Fuchs J, Schubert V, Peska V, Desvoyes B, Gutierrez C, Fajkus J (2018) Replication of ribosomal DNA in Arabidopsis occurs both inside and outside the nucleolus during S phase progression. J Cell Sci $\mathbf{1 3 1}$

Earley KW, Pontvianne F, Wierzbicki AT, Blevins T, Tucker S, Costa-Nunes P, Pontes O, Pikaard CS (2010) Mechanisms of HDA6-mediated rRNA gene silencing: suppression of intergenic Pol II transcription and differential effects on maintenance versus siRNA-directed cytosine methylation. Genes \& development 24: $1119-1132$

Franek M, Kovarikova A, Bartova E, Kozubek S (2016) Nucleolar Reorganization Upon Site-Specific Double-Strand Break Induction. The journal of histochemistry and cytochemistry : official journal of the Histochemistry Society 64: 669-686

Fransz P, De Jong JH, Lysak M, Castiglione MR, Schubert I (2002) Interphase chromosomes in Arabidopsis are organized as well defined chromocenters from which euchromatin loops emanate. Proceedings of the National Academy of Sciences of the United States of America 99: 14584-14589 
Fransz P, ten Hoopen R, Tessadori F (2006) Composition and formation of heterochromatin in Arabidopsis thaliana. Chromosome research : an international journal on the molecular, supramolecular and evolutionary aspects of chromosome biology 14: 71-82

Gordon GW, Berry G, Liang XH, Levine B, Herman B (1998) Quantitative fluorescence resonance energy transfer measurements using fluorescence microscopy. Biophysical journal 74: 2702-2713

Granot G, Sikron-Persi N, Li Y, Grafi G (2009) Phosphorylated H3S10 occurs in distinct regions of the nucleolus in differentiated leaf cells. Biochimica et biophysica acta 1789: 220-224

Harding SM, Boiarsky JA, Greenberg RA (2015) ATM Dependent Silencing Links Nucleolar Chromatin Reorganization to DNA Damage Recognition. Cell reports 13: 251-259

Highett MI, Rawlins DJ, Shaw PJ (1993) Different Patterns of Rdna Distribution in Pisum-Sativum Nucleoli Correlate with Different Levels of Nucleolar Activity. Journal of cell science 104: 843-852

Huang J, Ma L, Yang F, Fei SZ, Li L (2008) 45S rDNA regions are chromosome fragile sites expressed as gaps in vitro on metaphase chromosomes of root-tip meristematic cells in Lolium spp. PLoS One 3: e2167

Chandrasekhara C, Mohannath G, Blevins T, Pontvianne F, Pikaard CS (2016) Chromosome-specific NOR inactivation explains selective rRNA gene silencing and dosage control in Arabidopsis. Genes \& development 30: $177-190$

Chen ZJ, Comai L, Pikaard CS (1998) Gene dosage and stochastic effects determine the severity and direction of uniparental ribosomal RNA gene silencing (nucleolar dominance) in Arabidopsis allopolyploids. Proceedings of the National Academy of Sciences of the United States of America 95: 14891-14896

Chen ZJ, Pikaard CS (1997) Epigenetic silencing of RNA polymerase I transcription: a role for DNA methylation and histone modification in nucleolar dominance. Genes \& development 11: 2124-2136

Chica C, Szarzynska B, Chen-Min-Tao R, Duvernois-Berthet E, Kassam M, Colot V, Roudier F (2013) Profiling spatial enrichment of chromatin marks suggests an additional epigenomic dimension in gene regulation. Front Life Sci 7: 80-87

Jackson SA, Wang ML, Goodman HM, Jiang J (1998) Application of fiber-FISH in physical mapping of Arabidopsis thaliana. Genome 41: 566-572

Jacob Y, Michaels SD (2009) H3K27me1 is E(z) in animals, but not in plants. Epigenetics 4: 366-369

Lafontaine JG (1958) Structure and mode of formation of the nucleolus in meristematic cells of Vicia faba and Allium cepa. The Journal of biophysical and biochemical cytology 4: 777-784

Lawrence RJ, Earley K, Pontes O, Silva M, Chen ZJ, Neves N, Viegas W, Pikaard CS (2004) A concerted DNA methylation/histone methylation switch regulates rRNA gene dosage control and nucleolar dominance. Molecular cell 13: 599-609

Leitch AR, Mosgoller W, Shi M, Heslop-Harrison JS (1992) Different patterns of rDNA organization at interphase in nuclei of wheat and rye. Journal of cell science 101 ( Pt 4): 751-757

Li J, Langst G, Grummt I (2006) NoRC-dependent nucleosome positioning silences rRNA genes. The EMBO journal 25: 5735-5741 
Liang YM, Wang X, Ramalingam R, So KY, Lam YW, Li ZF (2012) Novel nucleolar isolation method reveals rapid response of human nucleolar proteomes to serum stimulation. J Proteomics 77: 521-530

Liu N, Fromm M, Avramova Z (2014) H3K27me3 and H3K4me3 chromatin environment at super-induced dehydration stress memory genes of Arabidopsis thaliana. Molecular plant 7: 502-513

Mandakova T, Lysak MA (2008) Chromosomal phylogeny and karyotype evolution in $\mathrm{x}=7$ crucifer species (Brassicaceae). The Plant cell 20: 2559-2570

Mathieu O, Jasencakova Z, Vaillant I, Gendrel AV, Colot V, Schubert I, Tourmente S (2003) Changes in 5S rDNA chromatin organization and transcription during heterochromatin establishment in Arabidopsis. The Plant cell 15: 2929-2939

Mathieu O, Probst AV, Paszkowski J (2005) Distinct regulation of histone H3 methylation at lysines 27 and 9 by CpG methylation in Arabidopsis. The EMBO journal 24: 2783-2791

McKeown PC, Shaw PJ (2009) Chromatin: linking structure and function in the nucleolus. Chromosoma 118: $11-23$

Medina FJ, Cerdido A, de Carcer G (2000) The functional organization of the nucleolus in proliferating plant cells. European journal of histochemistry : EJH 44: 117-131

Melcak I, Risueno MC, Raska I (1996) Ultrastructural nonisotopic mapping of nucleolar transcription sites in onion protoplasts. Journal of structural biology 116: 253-263

Mohannath G, Pontvianne F, Pikaard CS (2016) Selective nucleolus organizer inactivation in Arabidopsis is a chromosome position-effect phenomenon. Proceedings of the National Academy of Sciences of the United States of America 113: 13426-13431

Moissiard G, Cokus SJ, Cary J, Feng S, Billi AC, Stroud H, Husmann D, Zhan Y, Lajoie BR, McCord RP, Hale CJ, Feng W, Michaels SD, Frand AR, Pellegrini M, Dekker J, Kim JK, Jacobsen SE (2012) MORC family ATPases required for heterochromatin condensation and gene silencing. Science 336: 1448-1451

Montacie C, Durut N, Opsomer A, Palm D, Comella P, Picart C, Carpentier MC, Pontvianne F, Carapito C, Schleiff E, Saez-Vasquez J (2017) Nucleolar Proteome Analysis and Proteasomal Activity Assays Reveal a Link between Nucleolus and 26S Proteasome in A. thaliana. Frontiers in plant science 8: 1815

Mozgova I, Mokros P, Fajkus J (2010) Dysfunction of chromatin assembly factor 1 induces shortening of telomeres and loss of 45S rDNA in Arabidopsis thaliana. Plant Cell 22: 2768-2780

Neves N, Viegas W, Pikaard CS (2005) Nucleolar dominance and rRNA gene dosage control: a pardigm for transcriptional regulation via an epigentic on/off switch. Annual Plant Reviews 19: 201-222

Nie X, Wang H, Li J, Holec S, Berger F (2014) The HIRA complex that deposits the histone H3.3 is conserved in Arabidopsis and facilitates transcriptional dynamics. Biol Open 3: 794-802

Otero S, Desvoyes B, Peiro R, Gutierrez C (2016) Histone H3 Dynamics Reveal Domains with Distinct Proliferation Potential in the Arabidopsis Root. Plant Cell 28: 1361-1371

Pavlistova V, Dvorackova M, Jez M, Mozgova I, Mokros P, Fajkus J (2016) Phenotypic reversion in fas mutants of Arabidopsis thaliana by reintroduction of FAS genes: variable recovery of telomeres with major spatial rearrangements and transcriptional reprogramming of 45S rDNA genes. Plant J 88: 411-424 
Pendle AF, Clark GP, Boon R, Lewandowska D, Lam YW, Andersen J, Mann M, Lamond AI, Brown JW, Shaw PJ (2005) Proteomic analysis of the Arabidopsis nucleolus suggests novel nucleolar functions. Molecular biology of the cell 16: 260-269

Perry RP (1966) Nucleolus: structure and function. Science 153: 214-219

Pontes O, Lawrence RJ, Silva M, Preuss S, Costa-Nunes P, Earley K, Neves N, Viegas W, Pikaard CS (2007) Postembryonic establishment of megabase-scale gene silencing in nucleolar dominance. PloS one 2: e1157

Pontvianne F, Abou-Ellail M, Douet J, Comella P, Matia I, Chandrasekhara C, Debures A, Blevins T, Cooke R, Medina FJ, Tourmente S, Pikaard CS, Saez-Vasquez J (2010) Nucleolin is required for DNA methylation state and the expression of rRNA gene variants in Arabidopsis thaliana. PLoS genetics 6: e1001225

Pontvianne F, Blevins T, Chandrasekhara C, Feng W, Stroud H, Jacobsen SE, Michaels SD, Pikaard CS (2012) Histone methyltransferases regulating rRNA gene dose and dosage control in Arabidopsis. Genes \& development 26: $945-957$

Pontvianne F, Blevins T, Chandrasekhara C, Mozgova I, Hassel C, Pontes OM, Tucker S, Mokros P, Muchova V, Fajkus J, Pikaard CS (2013) Subnuclear partitioning of rRNA genes between the nucleolus and nucleoplasm reflects alternative epiallelic states. Genes Dev 27: 1545-1550

Probst AV, Fagard M, Proux F, Mourrain P, Boutet S, Earley K, Lawrence RJ, Pikaard CS, Murfett J, Furner I, Vaucheret H, Mittelsten Scheid O (2004) Arabidopsis histone deacetylase HDA6 is required for maintenance of transcriptional gene silencing and determines nuclear organization of rDNA repeats. Plant Cell 16: $1021-1034$

Pruitt RE, Meyerowitz EM (1986) Characterization of the genome of Arabidopsis thaliana. Journal of molecular biology 187: 169-183

Risueno MC, Medina FJ, Moreno Diaz de la Espina S (1982) Nucleolar fibrillar centres in plant meristematic cells: ultrastructure, cytochemistry and autoradiography. Journal of cell science 58: 313-329

Roudier F, Ahmed I, Berard C, Sarazin A, Mary-Huard T, Cortijo S, Bouyer D, Caillieux E, DuvernoisBerthet E, Al-Shikhley L, Giraut L, Despres B, Drevensek S, Barneche F, Derozier S, Brunaud V, Aubourg S, Schnittger A, Bowler C, Martin-Magniette ML, Robin S, Caboche M, Colot V (2011) Integrative epigenomic mapping defines four main chromatin states in Arabidopsis. EMBO J 30: 1928-1938

Sequeira-Mendes J, Araguez I, Peiro R, Mendez-Giraldez R, Zhang X, Jacobsen SE, Bastolla U, Gutierrez C (2014) The Functional Topography of the Arabidopsis Genome Is Organized in a Reduced Number of Linear Motifs of Chromatin States. Plant Cell 26: 2351-2366

Shaw PJ, Jordan EG (1995) The nucleolus. Annual review of cell and developmental biology 11: 93-121

Shi L, Wang J, Hong F, Spector DL, Fang Y (2011) Four amino acids guide the assembly or disassembly of Arabidopsis histone H3.3-containing nucleosomes. Proceedings of the National Academy of Sciences of the United States of America 108: 10574-10578

Schofer C, Weipoltshammer K (2018) Nucleolus and chromatin. Histochemistry and cell biology 150: 209225

Schwartz YB, Pirrotta V (2007) Polycomb silencing mechanisms and the management of genomic programmes. Nature reviews Genetics 8: 9-22 
Smirnov E, Borkovec J, Kovacik L, Svidenska S, Schrofel A, Skalnikova M, Svindrych Z, Krizek P, Ovesny M, Hagen GM, Juda P, Michalova K, Cardoso MC, Cmarko D, Raska I (2014) Separation of replication and transcription domains in nucleoli. Journal of structural biology 188: 259-266

Smith S, Stillman B (1989) Purification and characterization of CAF-I, a human cell factor required for chromatin assembly during DNA replication in vitro. Cell 58: $15-25$

Soppe WJ, Jasencakova Z, Houben A, Kakutani T, Meister A, Huang MS, Jacobsen SE, Schubert I, Fransz PF (2002) DNA methylation controls histone H3 lysine 9 methylation and heterochromatin assembly in Arabidopsis. The EMBO journal 21: 6549-6559

Stepinski D (2014) Functional ultrastructure of the plant nucleolus. Protoplasma 251: 1285-1306

Stroud H, Otero S, Desvoyes B, Ramirez-Parra E, Jacobsen SE, Gutierrez C (2012) Genome-wide analysis of histone H3.1 and H3.3 variants in Arabidopsis thaliana. Proceedings of the National Academy of Sciences of the United States of America 109: 5370-5375

Tagami H, Ray-Gallet D, Almouzni G, Nakatani Y (2004) Histone H3.1 and H3.3 complexes mediate nucleosome assembly pathways dependent or independent of DNA synthesis. Cell 116: 51-61

Tessadori F, Chupeau MC, Chupeau Y, Knip M, Germann S, van Driel R, Fransz P, Gaudin V (2007) Largescale dissociation and sequential reassembly of pericentric heterochromatin in dedifferentiated Arabidopsis cells. Journal of cell science 120: $1200-1208$

Thorstensen T, Fischer A, Sandvik SV, Johnsen SS, Grini PE, Reuter G, Aalen RB (2006) The Arabidopsis SUVR4 protein is a nucleolar histone methyltransferase with preference for monomethylated H3K9. Nucleic acids research 34: 5461-5470

van Sluis M, McStay B (2019) Nucleolar DNA Double-Strand Break Responses Underpinning rDNA Genomic Stability. Trends in genetics : TIG 35: 743-753

Vaquero-Sedas MI, Luo C, Vega-Palas MA (2012) Analysis of the epigenetic status of telomeres by using ChIP-seq data. Nucleic acids research 40: e163

Watanabe-Susaki K, Takada H, Enomoto K, Miwata K, Ishimine H, Intoh A, Ohtaka M, Nakanishi M, Sugino H, Asashima M, Kurisaki A (2014) Biosynthesis of ribosomal RNA in nucleoli regulates pluripotency and differentiation ability of pluripotent stem cells. Stem cells 32: 3099-3111

Wollmann H, Stroud H, Yelagandula R, Tarutani Y, Jiang D, Jing L, Jamge B, Takeuchi H, Holec S, Nie X, Kakutani T, Jacobsen SE, Berger F (2017) The histone H3 variant H3.3 regulates gene body DNA methylation in Arabidopsis thaliana. Genome Biol 18: 94

Yelagandula R, Stroud H, Holec S, Zhou K, Feng S, Zhong X, Muthurajan UM, Nie X, Kawashima T, Groth M, Luger K, Jacobsen SE, Berger F (2014) The histone variant H2A.W defines heterochromatin and promotes chromatin condensation in Arabidopsis. Cell 158: 98-109

Zentner GE, Henikoff S (2014) High-resolution digital profiling of the epigenome. Nature reviews Genetics 15: $814-827$

Zentner GE, Saiakhova A, Manaenkov P, Adams MD, Scacheri PC (2011) Integrative genomic analysis of human ribosomal DNA. Nucleic acids research 39: 4949-4960

Zhao Z, Dammert MA, Grummt I, Bierhoff H (2016) lncRNA-Induced Nucleosome Repositioning Reinforces Transcriptional Repression of rRNA Genes upon Hypotonic Stress. Cell reports 14: 1876-1882 


\section{Figure legends}

Fig. 1. Composition of nucleolar foci (NF) and perinucleolar chromocenters (PNCs).

(A) Distribution of histone H3 variants H3.1 and H3.3 in both PNCs and NF of RedGreen (H3.1-GFP; H3.3mRFP) and GreenRed (H3.1-mRFP; H3.3-GFP) transgenic lines. Seedlings were imaged on a confocal microscope, H3.1 always color-coded green and H3.3 red for clarity. (B) Quantification of the number of monovariant (H3.3-only) and bivariant (H3.1 + H3.3) NF and PNCs observed in the nuclei of both plant lines. A minority of nuclei do not show any nucleolar foci. (C) FLIM-FRET experiment showing donor average fluorescence lifetimes $(\tau)$ for control (H3.1-GFP) and RedGreen plant lines. Box plot shows the data of the control sample. Each point belonging to RedGreen sample corresponds to a single FLIM-FRET measurement. The GFP average lifetime (y-axis) in RedGreen plants decreases with growing AOD (shown as blue triangle). (D) IF-FISH on nucleoli isolated from Myc-tagged lines showing colocalisation of H3.1 (green) and H3.3 (green) with rDNA (magenta). Images were acquired using STED microscopy (enhanced spatial resolution for green + magenta channels). ( $\mathrm{E}$ and $\mathrm{F}$ ) Examples of line profiles displaying various degree of overlap between H3.3 variant and rDNA (1 - strong overlap; 2 - partial overlap; 3 - no overlap in small rDNA foci). Scale bar $-2 \mu \mathrm{m}$. ERZ - endoreduplication zone; NF - nucleolar foci; PNCs perinucleolar chromocenters; ROI - region of interest; AOD - acceptor over donor ratio; RFI - relative fluorescence intensity.

Fig. 2. Organization of nucleolar foci (NF) and perinucleolar chromocenters (PNCs) in low-copy lines.

(A and B) Analysis of histone H3.3 foci in the roots of H3.3-GFP transformed wild-type (WT) and low-copy rDNA (L9) plants by confocal microscopy. Data shown in (B) were counted from 22 images of WT roots and 17 Images of low-copy roots (3 roots per sample). (C) Organization of rDNA in the roots of WT and low-copy rDNA (L6) line using whole-mount rDNA FISH (DAPI - gray, rDNA - magenta), wide-field microscopy combined with deconvolution. (D and E) Analysis of rDNA ultrastructure using STED, rDNA (magenta), DAPI (grey, image E), merged image (D). (D1-D3) Small rDNA foci, (D4-D6) larger clusters of rDNA together with dispersed rDNA signals, (D7-D9) compact foci without dispersed rDNA signals. Scale bars $-5 \mu \mathrm{m}$ (A and $\mathrm{C}) ; 2 \mu \mathrm{m}$ (D and $\mathrm{E})$.

Fig. 3. Distribution of H3.1 and H3.3 in the rDNA repeat based on ChiP-seq.

(A and B) Schematic depiction of a rDNA unit used for mapping with H3.1 and H3.3 distribution profiles in (A) actively dividing (div) and (B) non-dividing (ndiv) tissues of WT plants. (C and D) Schematic depiction of mappable part of (C) rDNA promoter region and (D) rDNA TTS with profiles of H3.1 and H3.3 in dividing and non-dividing tissues (enlarged from A and B). (E) Schematic depiction of a rDNA unit with H3 histone distribution profiles in wild type (WT) and low rDNA copy line (L9). (F and G) Schematic depiction of mappable part of (F) rDNA promoter region and (G) rDNA TTS with H3 distribution profiles from WT and L9 (enlarged from (E)). ETS - external transcribed spacer; LMR - low mappability region; TSS transcription start site.

Fig. 4. Distribution of epigenetic modifications in the rDNA.

(A and B) Schematic depiction of a rDNA unit with ChIP-seq profiles of (A) H3K4me3, (B) H3K27me3, (C) H2A.W and (D) H3K9me2 in rDNA created from publicly available datasets. ChIP experiments with subsequent dot-blot signal detection showing levels of $\mathrm{H} 3$ histone and corresponding epigenetic marks at the (E) 18S rDNA and (F) TSS regions of a rDNA unit in WT and low rDNA copy lines L6 and L9. (G) Bar plot evaluation of $\mathrm{H} 3 \mathrm{~K} 9 \mathrm{me} 2$ immunoprecipitation efficiency calculated from $\mathrm{E}$ and $\mathrm{F}$ experiments. ETS external transcribed spacer; LMR - low mappability region; TSS - transcription start site. 
Fig. 5. Distribution patterns of H3K9me2; H3K27me3 and H3K4m3 in rDNA based on advanced microscopy methods (A,G - confocal, C,D,E,F - dSTORM, H - SIM)

(A) H3K9me2 pattern in nuclear chromocenters and nucleolar foci (NF). NF (indicated by arrows) showing either accumulation of H3K9me2 $(1,2)$ or lack of H3K9me2 signal $(3,4)$. (B) Percentage of nuclei with NF labelled by H3K9me2 in WT and low-copy line L9. (C-F) Images of nuclei obtained by dSTORM microscopy, insets show a more detailed view of the nucleolus. Distribution of H3K4me3 (C) and H3K27me3 (D) in plant nuclei. (E and F) Mutual distribution of H3K4me3 and H3K27me3 in the nucleoli of WT and low-copy plant lines. ( $\mathrm{G}$ and $\mathrm{H}$ ) Distribution of H3K4me3 (G5 and G6; H5 and H6), H3K27me3 (G3 and G4) and H3K9me2 (H3 and H4) in clusters of rDNA (G1 and G2; H1 and H2) visualized on chromatin fibers. (G7 and G8) Overlay images showing partial colocalisation between H3K4me3 and H3K27me3. (H7) Lack of colocalisation between H3K4me3 and H3K9me2 histone marks. (H8) Segments of rDNA clusters largely devoid of repressive marks. Scale bars (A) - $5 \mu \mathrm{m}$. Scale bars $(\mathrm{C}-\mathrm{H})-2 \mu \mathrm{m}$. NF - nucleolar foci. 


\section{Figures}

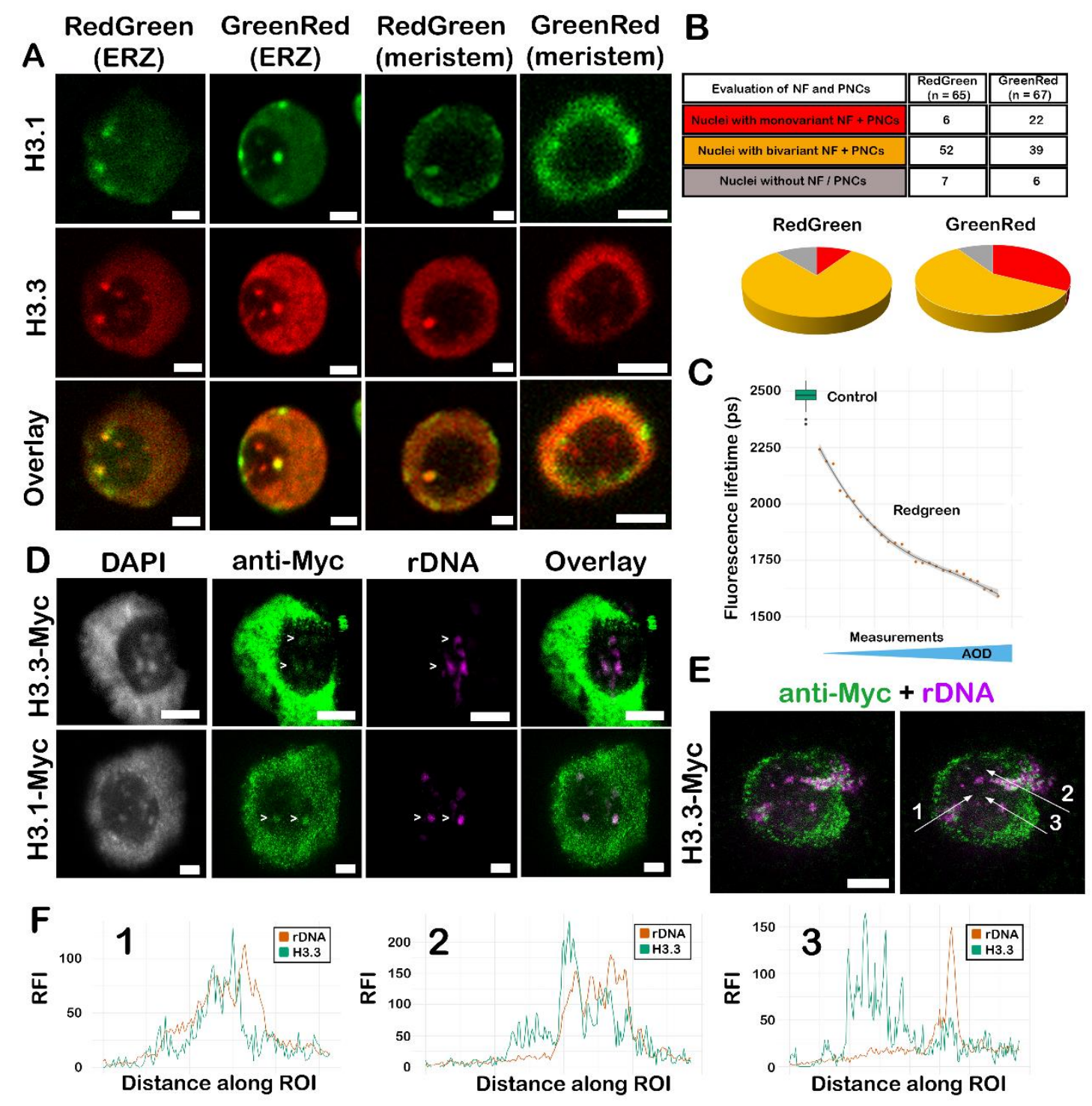

Fig. 1. Composition of nucleolar foci (NF) and perinucleolar chromocenters (PNCs). 

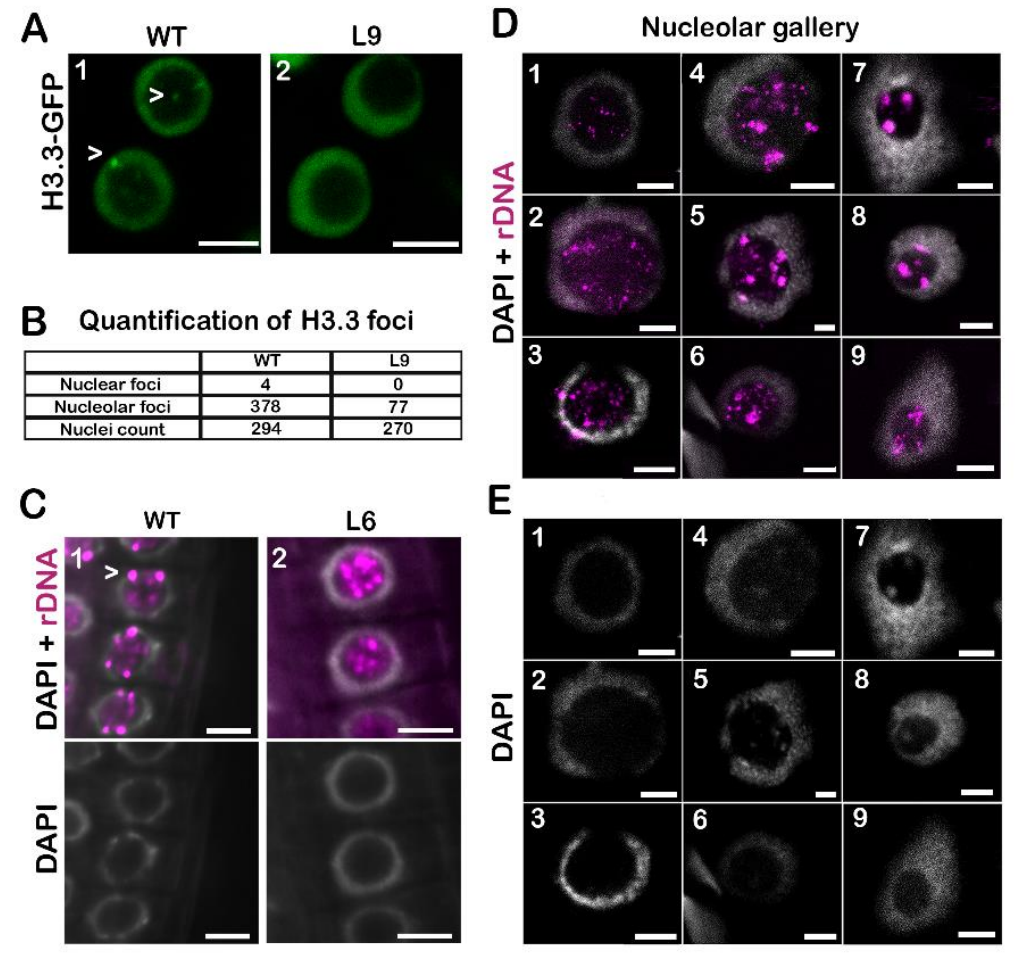

Fig. 2. Organization of nucleolar foci (NF) and perinucleolar chromocenters (PNCs) in low-copy lines. 


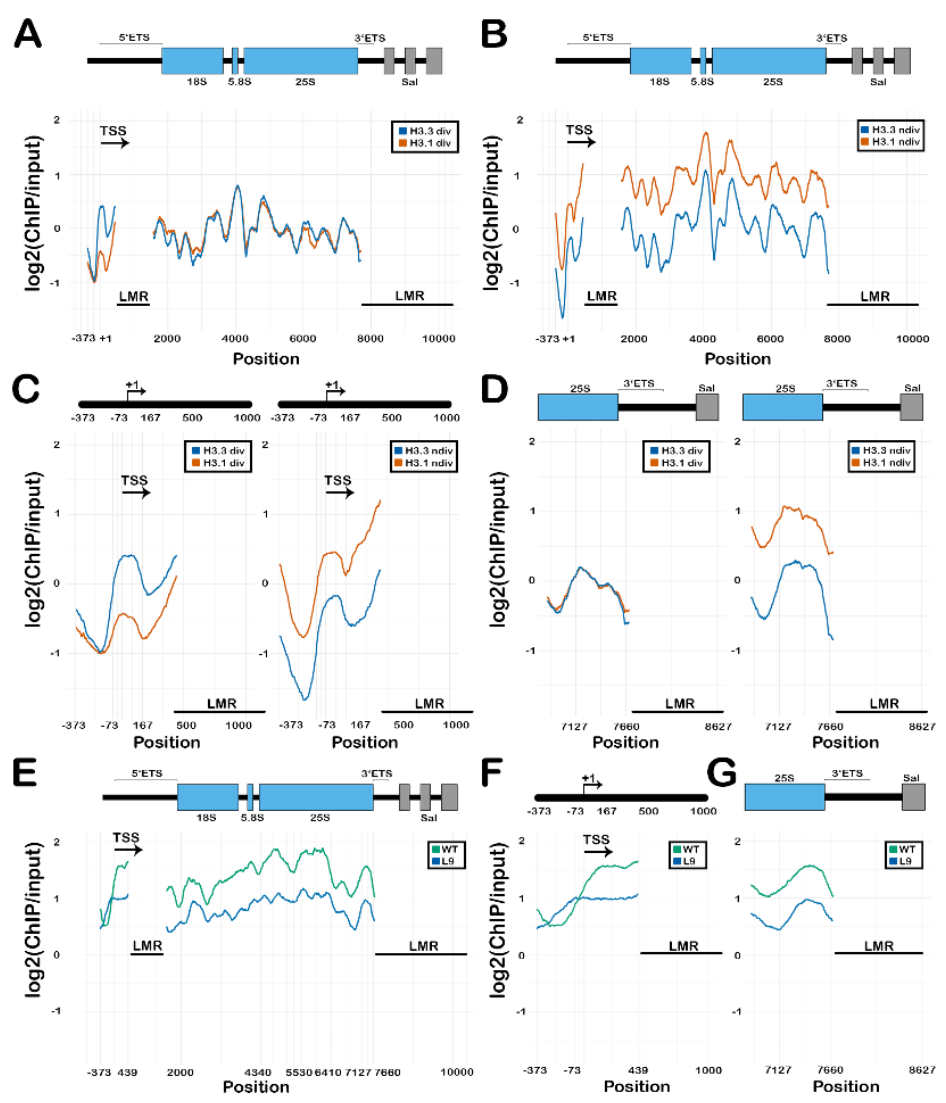

Fig. 3. Distribution of H3.1 and H3.3 in the rDNA repeat based on ChiP-seq. 
A
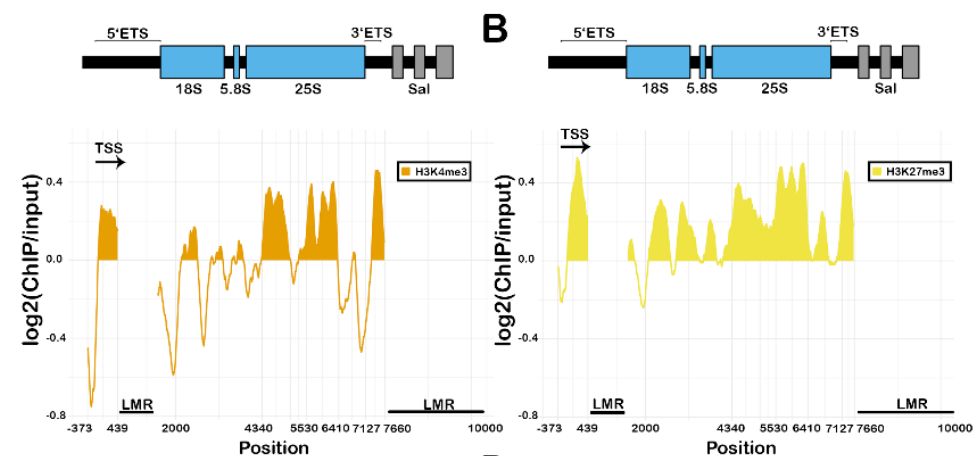

C

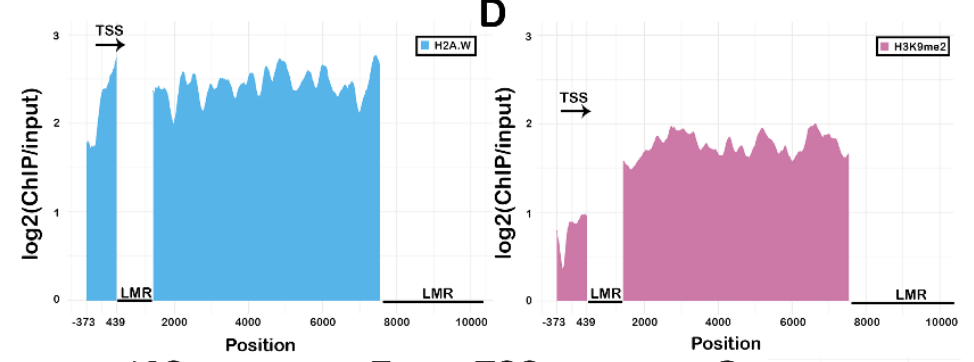

E

$18 \mathrm{~S}$

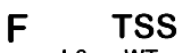

G H3K9me2

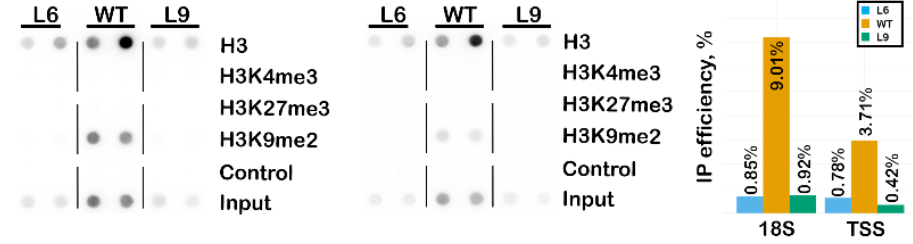

Fig. 4. Distribution of epigenetic modifications in the rDNA. 
A
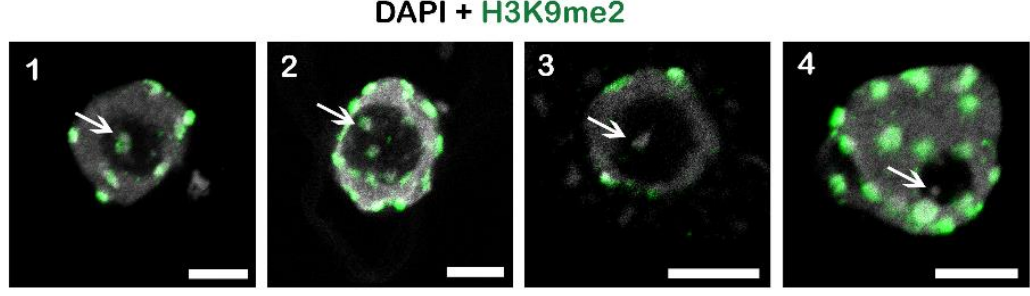

B

\begin{tabular}{|c|c|} 
H3K9me2 labelled NF \\
\hline WT & L6 \\
\hline \hline $22 / 44$ & $8 / 32$ \\
\hline \hline $50 \%$ & $25 \%$ \\
\hline
\end{tabular}
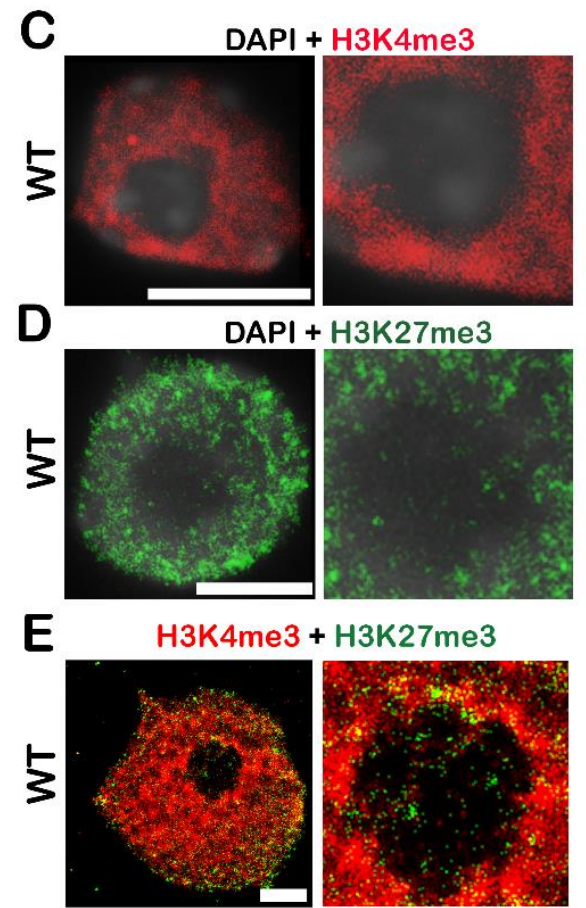

$\mathbf{F}$
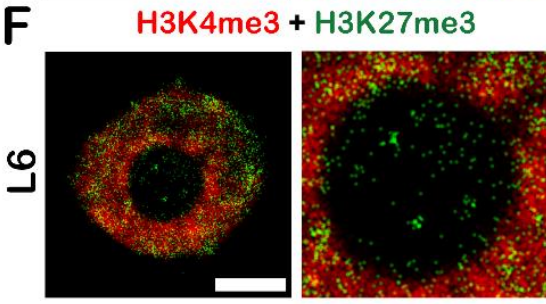

G
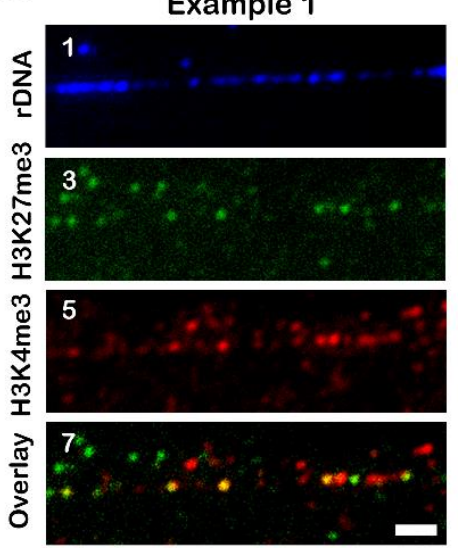

$\mathrm{H}$

Example 3
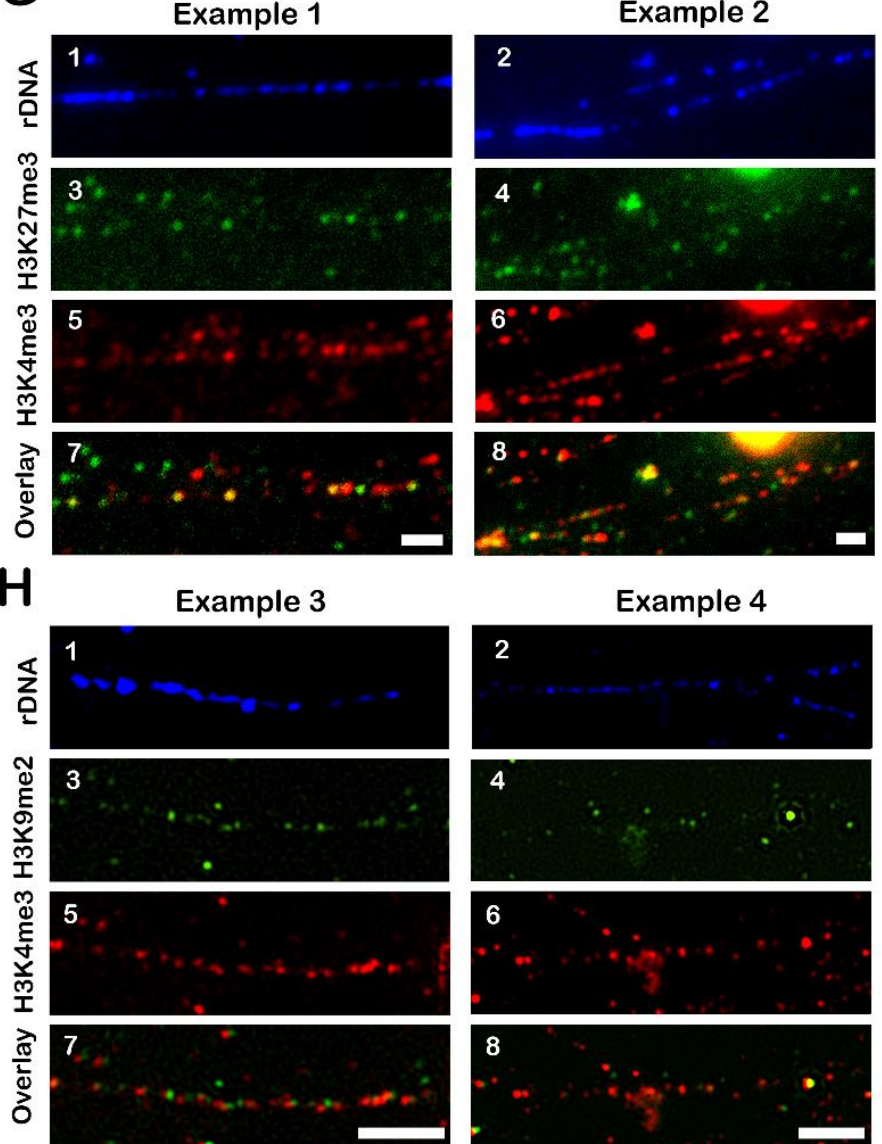

Example 4
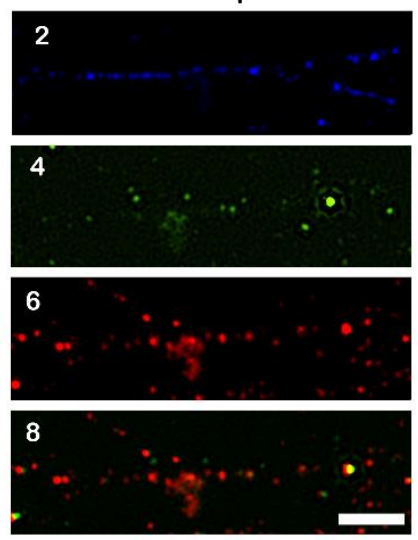

Fig. 5. Distribution patterns of $\mathrm{H} 3 \mathrm{~K} 9 \mathrm{me} 2 ; \mathrm{H} 3 \mathrm{~K} 27 \mathrm{me} 3$ and $\mathrm{H} 3 \mathrm{~K} 4 \mathrm{~m} 3$ in rDNA based on advanced microscopy methods (A,G - confocal, C,D,E,F - dSTORM, H - SIM) 


\section{Supplementary information}

\section{Supplementary material}

\section{Plant material}

All $A$. thaliana lines used in this study were derived from the Columbia ecotype. Previously described lines were used: fas1-4 (At1g65470) (NASC: N828822, SAIL 662_D10 (Exner et al, 2006; Mozgova et al, 2010); plant lines transformed with HTR3-GFP, HTR4-GFP, HTR3-Myc, HTR4-Myc, HTR13-mRFP and double transformants HTR5-mRFP/HTR3-GFP ("RedGreen") were provided by C. Gutierrez CBMSO, Madrid, Spain (Otero et al, 2016; Stroud et al, 2012); fibrillarin-YFP plant line was obtained from F. Pontvianne, CNRS, Plant Genome and Development, Perpignan, France. Plant lines with reduced rDNA amount - line 6 (L6) and 9 (L9) were previously obtained and characterised in our laboratory (Pavlistova et al, 2016).

In A.thaliana, H3 variants are encoded by several HISTONE 3 RELATED (HTR) genes, namely HTR1, HTR2, HTR3, HTR9 and HTR13 for H3.1 and HTR4, HTR5 and HTR8 encoding H3.3. We used HTR4 (At4g40030) and HTR5 (At4g40040) for H3.3 and HTR3 (At3g27360) and HTR13 (At5g10390) for H3.1. Plant line carrying both HTR4-GFP and HTR13-mRFP ("GreenRed") fusion proteins was obtained by crossing of HTR4-GFP and HTR13-mRFP plants and selection of double labelled plants in F2. HTR4-GFP + L9 were prepared by crossing the fas 1-4 expressing HTR4-GFP (Otero et al, 2016) into line L9 background (Pavlistova et al, 2016).

\section{Supplementary methods}

\section{Plant growth}

All seeds were sterilized (70\% ethanol/ $10 \mathrm{~min}$ followed by $99 \%$ ethanol $5 \mathrm{~min}$ ) and plated on half-strength agar Murashige and Skoog medium ( $1 \frac{1}{2}$ MS medium) with $1 \%$ sucrose. After 2 -day stratification $\left(4^{\circ} \mathrm{C} /\right.$ dark $)$ plates were transferred to the growth chamber and pre-grown up to 2 weeks under long day (LD) conditions (16 h light $-21^{\circ} \mathrm{C} / 8 \mathrm{~h}$ dark $-19^{\circ} \mathrm{C} / 50-60 \%$ relative humidity). Seedlings were then collected directly from plates and used for experiments or transferred into the soil and grown under the same long day conditions or transferred to the short day $\left(8 \mathrm{~h}\right.$ light $-21^{\circ} \mathrm{C} / 16 \mathrm{~h}$ dark $-19^{\circ} \mathrm{C} / 50-60 \%$ relative humidity).

\section{Isolation of unfixed nuclei for fiber preparations}

Twelve-d-old seedlings ( $0.5 \mathrm{~g}$ ) were chopped with the razor blade in ice-cold nucleus isolation buffer (NIB $0.5 \mathrm{M}$ sucrose; $10 \mathrm{~mm}$ EDTA; $2.5 \mathrm{~mm}$ DTT; $100 \mathrm{mM} \mathrm{KCl} ; 1 \mathrm{mM}$ spermine; $4 \mathrm{~mm}$ spermidine in $10 \mathrm{~mm}$ Tris$\mathrm{Cl}, \mathrm{pH}$ 9.5). After chopping, the solution was filtered through $50 \mu \mathrm{m}$ and $30 \mu \mathrm{m}$ pore size filters (CellTrics, Sysmex, Germany). Afterwards, the filtrate was supplemented with $1 / 10$ volume of $10 \%$ Triton-X in NIB and centrifuged at $2000 \mathrm{~g}$ for $10 \mathrm{~min}$ at $4^{\circ} \mathrm{C}$. The pelleted nuclei were then resuspended in $400 \mu \mathrm{L}$ of NIB and supplemented with $400 \mu \mathrm{L}$ of $100 \%$ glycerol. Nuclei were then aliquoted and stored at $-20^{\circ} \mathrm{C}$.

\section{Isolation of nucleoli}

Nucleoli from 10-d-old seedlings were isolated based on the published protocol (Liang et al, 2012) with some modifications. Seedlings were fixed in 1\% paraformaldehyde (PFA) for 10 min then quenched with $0.125 \mathrm{M}$ glycine for $5 \mathrm{~min}$ (both under vacuum). Seedlings were disrupted in $20 \mathrm{~mL}$ of Galbraith buffer, (GB), without Triton X (Galbraith et al, 2011) using ICA T25 Ultra Turrax homogenizer (11000 rpm, $90 \mathrm{~s})$ and filtered through $40 \mu \mathrm{m}$ nylon cell strainer filters (Falcon, \#352340). Then Triton-X was added to the final concentration of $0.3 \%$. Homogenate was vortexed, left $5 \mathrm{~min}$ on ice and centrifuged at $350 \mathrm{~g}$ for $20 \mathrm{~min}$ at $4^{\circ} \mathrm{C}$. Pellet was resuspended in Solution I (Liang et al, 2012) $\left(0.5 \mathrm{M}\right.$ sucrose with $3 \mathrm{mM} \mathrm{MgCl}_{2}$ and protease inhibitors), $500 \mu \mathrm{L}$ aliquots were used for sonication (Diagenode Bioruptor; 5 x 5 min cycles of $30 \mathrm{~s}$ ON / 30 s OFF at $\mathrm{H}$ mode). Sonicated nuclei were underlaid with $700 \mu \mathrm{L}$ of Solution II (1 M sucrose, $3 \mathrm{mM} \mathrm{MgCl}_{2}$ with protease inhibitors) and centrifuged at $1800 \mathrm{~g}$ for $5 \mathrm{~min}$ at $4{ }^{\circ} \mathrm{C}$. Pellet containing nucleoli was resuspended in $500 \mu \mathrm{L}$ of storage buffer ( $90 \%$ Glycerol, $10 \% \mathrm{~GB})$ and stored at $-20^{\circ} \mathrm{C}$. 


\section{Chromatin fiber preparation}

An aliquot of isolated nuclei was centrifuged at $5000 \mathrm{~g}$ for $10 \mathrm{~min}$ at $4{ }^{\circ} \mathrm{C}$. Then, nuclei were resuspended in $75 \mathrm{mM} \mathrm{KCl}$ (the dilution needs to be optimized for each isolation, for chromatin fibers we recommend first testing 1:50; 1:100 and 1:200 dilutions) and incubated for $20 \mathrm{~min} .8 \mu \mathrm{L}$ of the nuclear suspension were cytocentrifuged onto poly-L-lysine-coated coverslips at $400 \mathrm{~g}$ for $4 \mathrm{~min}$ at $4^{\circ} \mathrm{C}$. Coverslips were immediately removed from the centrifugation chambers, excess liquid was removed and $20 \mu \mathrm{L}$ of nucleus lysis buffer (NLB - $330 \mathrm{~mm} \mathrm{NaCl}, 500 \mathrm{mM}$ urea, $1 \%$ Triton-X 100, $25 \mathrm{~mm}$ Tris-Cl, $\mathrm{pH}=7$ ) were pipetted onto nuclei. The drop was quickly covered with a square parafilm coverslip $(15 \times 15 \mathrm{~mm}$ in size). NLB was left to evaporate for $60 \mathrm{~min}$, after which the coverslips were immersed in 1x KCM buffer $(120 \mathrm{mM} \mathrm{KCl}, 20 \mathrm{mM}$ $\mathrm{NaCl}, 10 \mathrm{~mm}$ Tris-Cl, $0.5 \mathrm{~mm}$ EDTA, $0.1 \%$ Triton-X) for 30 min. Blocking was performed with $5 \%$ BSA in $\mathrm{KCM}$ for $30 \mathrm{~min}$ at RT, after which the samples were incubated with primary antibodies diluted $1: 100$ in $5 \%$ BSA / KCM solution for $1 \mathrm{~h}$ at RT (anti-histone H3K4me3, ab8580; anti-histone H3K27me3, ab6002; antihistone H3K9me2, ab1220). Samples were washed three times for $5 \mathrm{~min}$ in KCM, then incubated with appropriate secondary antibodies diluted 1:200 in 5\% BSA / KCM. Samples were washed three times for 5 min in KCM, post-fixed in 4\% PFA in KCM, followed by two washes in 1x PBS. After mounting in DAPI + Vectashield, fiber stretching and immunofluorescence was checked on an epifluorescence microscope Zeiss Axioimager Z1. Antifade was then removed by two times 5 min washes in 1x PBS. Hybridisation mixture (50\% deionized formamide; $10 \%$ dextran sulfate; $1: 30$ diluted nick-translation labelled rDNA probe) was then pipetted onto clean glass slides and the coverslips with fibers were put face-down onto the glass slides and sealed off with rubber cement. Samples were denatured at $75^{\circ} \mathrm{C}$ for $3 \mathrm{~min}$ in a thermal cycler and hybridized $\mathrm{O} / \mathrm{N}$ at $37^{\circ} \mathrm{C}$. The next day, coverslips were washed once in $2 \mathrm{x} \mathrm{SSC}$ at $\mathrm{RT}$, then $3 \mathrm{x}$ in $50 \%$ formamide (FA) wash buffer $(50 \% \mathrm{FA}, 2 \mathrm{x} \mathrm{SSC})$ at $37^{\circ} \mathrm{C}$ and then once in $2 \mathrm{x} \mathrm{SSC}$ at $37^{\circ} \mathrm{C}$. Coverslips were mounted in DAPI (4',6-Diamidine-2'-phenylindole dihydrochloride), $2 \mu \mathrm{g} / \mathrm{mL}$ in Vectashield and imaged.

\section{Nick-translation rDNA labelling}

Probe labelling was adapted from (Mandakova \& Lysak, 2008) using $1 \mu \mathrm{g}$ of BAC as a template, isolated using Macherey Nagel NucleoBond ${ }^{\circledR}$ Xtra Midi. For visualization of the 45S rDNA loci the BAC clone T15P10 (GenBank AL095897/8) was used.

\section{Immunofuorescence (IF) and FISH on nuclei and nucleoli}

Half g of 10-d-old seedlings fixed in 4\% PFA for 10 mins, then rinsed in GB, see above and homogenized with a razor blade in GB. The homogenate was filtered through $50 \mu \mathrm{m}$ and $30 \mu \mathrm{m}$ pore-size filters (CellTrics, Sysmex, Germany). Nuclei were centrifuged for $20 \mathrm{~min}$ at $350 \mathrm{~g}$ at $4^{\circ} \mathrm{C}$, resuspended in $1 \mathrm{x}$ PBS and stopped onto slides. Nuclei were briefly dried at $4{ }^{\circ} \mathrm{C}$, then fixed in $4 \%$ PFA in $1 \mathrm{x}$ PBS / $0.5 \%$ Triton-X. Nuclei were rinsed three times in 1x PBS, blocked in 5\% BSA / $1 \mathrm{x}$ PBS for 30 min and incubated with anti-Myc antibody (M4439; Sigma Aldrich) diluted 1:100 in 5\% BSA/1x PBS for 1 hour. Nuclei were washed three times for 5 min in 1x PBST (0.05\% Tween-20), then incubated with an appropriate secondary antibody (1:200 dilution, $1 \mathrm{~h}$ incubation). Slides were washed three times for $5 \mathrm{~min}$ in $1 \mathrm{x}$ PBST, then dehydrated in ethanol series. FISH procedure was performed as described in (Dvorackova et al, 2018).

\section{Whole-mount FISH}

Five to ten seedlings (10-d-old) were fixed for $30 \mathrm{~min}$ in $5 \mathrm{ml}$ of fixative ( $1 \%$ formaldehyde, $10 \%$ DMSO, $0.5 \mathrm{mM}$ EGTA in 1x PBS). Seedlings were rinsed two times $10 \mathrm{~min}$ in $100 \%$ methanol and two times $10 \mathrm{~min}$ in $96 \%$ ethanol, transferred to a $1.5 \mathrm{ml}$ Eppendorf tube and left at $4^{\circ} \mathrm{C}$ overnight. All subsequent washes were performed in Eppendorf tubes containing $1 \mathrm{ml}$ of appropriate buffer. Seedlings were transferred to a new tube, washed two times for $5 \mathrm{~min}$ in $1 \mathrm{x}$ PBS $/ 0.1 \%$ Tween- 20 and three times for $5 \mathrm{~min}$ in $2 \mathrm{x}$ SSC. Seedlings were incubated for $30 \mathrm{~min}$ in the mix of 2x SSC and HB50, 1:1 (HB= hybridisation buffer, $10 \%$ dextran sulfate, $50 \%$ deionized formamide in $2 \mathrm{x} \mathrm{SSC}) ; 30 \mathrm{~min}$ in $\mathrm{HB} 50(1 \mathrm{ml})$ and $1-2$ hours $\left(37^{\circ} \mathrm{C}\right.$, shaking at 300 $\mathrm{rpm})$ in $100 \mu \mathrm{L}$ of the probe mix ( $5 \mu \mathrm{L}$ of AlexaFluor 594 labelled rDNA probe in HB50). Before the incubation in the probe mix, seedlings were briefly dried on the filter paper to avoid probe dilution and the tube was vortexed. Seedlings in the probe mix were denatured for $4 \mathrm{~min}$ at $90^{\circ} \mathrm{C}$ in a thermomixer, cooled on ice for $3 \mathrm{~min}$ and hybridized $\mathrm{O} / \mathrm{N}$ at $37^{\circ} \mathrm{C}$. All subsequent washes were performed in the thermomixer 
(shaking at $300 \mathrm{rpm}$ ): $2 \mathrm{x} 5 \mathrm{~min}$ in $50 \%$ formamide $/ 2 \mathrm{x} \mathrm{SSC}$ at $42^{\circ} \mathrm{C}$; $1 \mathrm{x} 5 \mathrm{~min}$ in $2 \mathrm{x} \mathrm{SSC}$ and $1 \mathrm{x} 5 \mathrm{~min}$ in $1 \mathrm{x}$ $\mathrm{PBS}$, both at $42^{\circ} \mathrm{C}$. Seedlings were then disentangled from one another in $1 \mathrm{x}$ PBS in a petri dish, put onto a microscopic slide and covered with DAPI, $2 \mu \mathrm{g} / \mathrm{mL}$ in Vectashield.

\section{Chromatin immunoprecipitation (IP) and high throughput sequencing}

Two grams of 10-d-old seedling were fixed in $40 \mathrm{ml}$ of $1 \%$ formaldehyde and quenched with $2.7 \mathrm{ml}$ of $2 \mathrm{M}$ glycine on ice, rinsed several times with cold $\mathrm{mQ}$ water, dried with paper towels and frozen in liquid nitrogen. After grinding in $\mathrm{N}_{2}$ powder was transferred in $15 \mathrm{ml}$ of Extraction buffer $(10 \mathrm{mM}$ Tris $\mathrm{HCl} \mathrm{pH} 8,10 \mathrm{mM}$ $\mathrm{MgCl} 2,1 \mathrm{M}$ Sucrose, $5 \mathrm{mM} \beta$-Mercaptoethanol, $0.1 \mathrm{mM}$ PMSF and protease inhibitors - PI -Pepstatin and Leupeptin at $1 \mu \mathrm{g} / \mathrm{ml}$ final concentration) and thoroughly mixed using vortex. Solution was then filtered through $40 \mu \mathrm{m}$ cell strainer (Falcon) and miracloth and spun down for $20 \mathrm{~min}$ (following centrifugation steps were performed at $1000 \mathrm{~g}$ and $4^{\circ} \mathrm{C}$ ). Pellet was resuspended in $5 \mathrm{ml}$ of Extraction buffer supplemented with $1 \%$ Triton X-100, transferred into $15 \mathrm{ml}$ tube and centrifuged for $10 \mathrm{~min}$. This procedure was repeated 5 times in total. Pellet was then resuspended in $5 \mathrm{ml}$ of Extraction buffer without sucrose, centrifuged for 10 min and resuspended in $300 \mu \mathrm{l}$ of nuclei lysis buffer $(50 \mathrm{mM}$ Tris $\mathrm{HCl} \mathrm{pH} 8,10 \mathrm{mM}$ EDTA, $5 \mathrm{mM} \beta-$ Mercaptoethanol, 1\% Triton X-100, 0.1 mM PMSF and PI). $10 \mu \mathrm{l}$ of Input 1 were taken at this step for further control of genomic DNA integrity. Samples were then sonicated at diagenode bioruptor sonicator: $4 \times 5 \mathrm{~min}$ cycle $(30 \mathrm{~s} \mathrm{ON} / 30 \mathrm{~s}$ OFF) with 4 min incubation on ice in between. Samples were then centrifuged four times at $20817 \mathrm{~g}$ for $10 \mathrm{~min}$ at $4^{\circ} \mathrm{C}$ and supernatant was collected into new tube each time. $20 \mu \mathrm{l}$ of Input 2 were taken afterwards from samples. Integrity of genomic DNA and efficiency of the sonication were checked using Inputs 1 and 2 on 1\% agarose gel. Sonication fragment length was optimised to 100-500 bp. Supernatant was further split into two tubes and diluted 10 times with dilution buffer (16.7 mM Tris $\mathrm{HCl} \mathrm{pH} 8,1.2 \mathrm{mM}$ EDTA, 1.1\% Triton X-100, $167 \mathrm{mM} \mathrm{NaCl}$ ). Chromatin was pre-cleared using protein A/G agarose beads (40 $\mu l$ per sample) that were washed with dilution buffer $\left(300 \mathrm{~g}\right.$ centrifugation at $\left.4^{\circ} \mathrm{C}\right)$ prior to incubation. Preclearing was performed overnight on a stirring wheel at $4^{\circ} \mathrm{C}$. Bead-antibody complexes were prepared simultaneously: protein $\mathrm{A} / \mathrm{G}$ agarose beads (40ul per immunoprecipitation reaction) were washed with dilution buffer and incubated with $2 \mu \mathrm{g}$ of antibody for 4 hours on a stirring wheel. Antibody-bead complexes were then blocked overnight in blocking buffer on a stirring wheel $(200 \mu \mathrm{g} / \mathrm{ml}$ glycogen, $3 \%$ BSA in dilution buffer). Pre-cleared samples were centrifuged at $1800 \mathrm{~g}$ for $5 \mathrm{~min}$ at $4^{\circ} \mathrm{C}$ and supernatant was split into two tubes (equal volume). 5\% of volume from each aliquote was collected together into a tube for further estimation of background (input sample). Antibody-bead complexes were washed twice with dilution buffer after blocking, mixed with samples and incubated overnight on a stirring wheel at $4^{\circ} \mathrm{C}$. Washing was performed using 4 buffers: wash buffer $1(50 \mathrm{mM}$ TrisHCl pH 8, $150 \mathrm{mM} \mathrm{NaCl}, 1 \%$ Igepal, 0.25\% Sodium deoxycholate, 1mM EDTA pH 8), wash buffer 2 (100 mM TrisHCl pH 8, $500 \mathrm{mM} \mathrm{LiCl,} \mathrm{1 \%} \mathrm{Igepal,} \mathrm{1 \%}$ Sodium deoxycholate), wash buffer 3 (100 mM TrisHCl pH 8, $150 \mathrm{mM} \mathrm{NaCl}, 500 \mathrm{mM} \mathrm{LiCl,} \mathrm{1 \%} \mathrm{Igepal,} \mathrm{1 \%}$ Sodium deoxycholate), and TE buffer according to the scheme: wash buffer 1 - wash 2 times, wash buffer $2-2$ times, wash buffer 3 - one time, TE - 2 times. Each wash was performed with 5 min incubation on stirring wheel in a cold room followed by $5 \mathrm{~min}$ centrifugation at $300 \mathrm{~g}$ at $4^{\circ} \mathrm{C}$. Tubes were changed at least twice during the washes. $250 \mu \mathrm{l}$ of elution buffer (1\% SDS 0,1M NaHCO3) were added to washed beads and they were incubated at thermomixer $\left(65^{\circ} \mathrm{C}, 1100 \mathrm{rpm}\right)$. Samples were centrifuged at $1800 \mathrm{~g}$ for $5 \mathrm{~min}$ and supernatant was transferred into a new tube. Elution procedure was then repeated and second supernatant was mixed with the first one. $20 \mu \mathrm{l}$ of $5 \mathrm{M} \mathrm{NaCl}$ were added to supernatant followed by incubation at $65^{\circ} \mathrm{C}$ overnight at $1100 \mathrm{rpm}$ on thermomixer. Input samples were added at this stage of protocol. Their volume was adjusted to $500 \mu \mathrm{l}$ using elution buffer and they were treated the same as immunoprecipitated samples. Samples were treated with RNAse by adding $20 \mu \mathrm{l}$ of TrisHCl pH 6.5 and $5 \mu \mathrm{l}$ of RNAse with incubation at $37^{\circ} \mathrm{C}$ for $30 \mathrm{~min}, 400 \mathrm{rpm}$. This was followed by proteinase $\mathrm{K}$ treatment by adding $10 \mu 1$ od $0.5 \mathrm{M}$ EDTA and $11.25 \mu \mathrm{l}$ of proteinase $\mathrm{K}$ with incubation for 2 hours at $45^{\circ} \mathrm{C}, 400 \mathrm{rpm} .11 .25 \mu \mathrm{l}$ of proteinase $\mathrm{K}$ were added afterwards with additional two-hour incubation under same conditions. Samples were precipitated by adding one tenth volume of sodium acetate $3 \mathrm{M}, \mathrm{pH} 4.8,1 \mu \mathrm{l}$ of glycogen and 3 volumes of EtOH. After thorough mixing samples were left at $-20{ }^{\circ} \mathrm{C}$ overnight. DNA was then pelleted by centrifugation at $20817 \mathrm{~g}$ in a precooled centrifuge for 1 hour. Pellet was then washed twice with cool $70 \%$ ethanol, each wash was followed by 15 min centrifugation at $20817 \mathrm{~g}, 4{ }^{\circ} \mathrm{C}$. Pellet was then air-dried and resuspended in $40 \mu 1$ of mQ water. All samples, except the first input were then cleaned using QIAquick PCR purification kit (Quiagen) according to instructions and eluted using $40 \mu \mathrm{l}$ of $\mathrm{mQ}$ water. Precipitated DNA was used for further analysis. 


\section{ChIP-seq data analysis}

Since ribosomal genes are not well annotated in the public databases, we first created a reference genome by merging the rDNA consensus sequence (Havlova et al, 2016) with TAIR10 genome. This sequence contained coding part of rDNA, 3' and 5' external transcribed spacer (ETS) sequences and non-transcribed spacer (NTS) and was merged with chromosome 2 sequence. Please note that there are some rDNA repeats already in TAIR10 genome, we performed N-masking of those using bedtools maskfasta command to avoid usage of those repeats and therefore loss of signal on our reference rDNA sequence.

We then evaluated the mappability of signals coming from different parts of rDNA sequence. Mappability is a measure of the uniqueness of a given genomic sequence based on the number of its fragments that are uniquely aligned to the particular sequence (Cheung et al, 2011; Zentner \& Henikoff, 2014). This analysis is necessary mostly because of NTS sequence that is not unique like the coding part and can have some homology to sequences outside of rDNA repeat. We used bias elimination algorithm for deep sequencing (BEADS) to calculate mappability values (Cheung et al, 2011). First rDNA repeat was sheared using GenomeTools shredder command into short sequences of $50 \mathrm{bp}$ length that correspond to lengths of ChIPseq reads and mapped them onto the constructed genome using bowtie 2 software. Mapped reads were extended to $200 \mathrm{bp}$ using beads extend command, as this length corresponds to the size of DNA associated with a single nucleosome. Mappability was calculated using beads tagCount command at for each base pair of our sequence (-base 1 option). Maximum mappability score with fragment length 200 can be 400 and it is considered as $100 \%$ mappability. Signal detected at this base pair is considered to be produced exclusively by rDNA. Base pairs with mappability lower than $25 \%$ are considered poorly mappable and should not be analysed since the signal could map from some other loci in the genome. Signals of low mappability were mainly localised in NTS and the highest mappability scores were at the promoter region and at the coding sequence (SI Appendix, Fig.S4 A).

ChIP-seq reads from our experiments and public datasets were aligned using bowtie 2 to previously described genome. Further processing was done using HOMER software (Heinz et al, 2010) according to the steps described in HOMER next generation sequencing analysis tutorial. Tag directories were constructed using makeTagDirectory command with selection of uniquely alignable reads (-unique option). Data for tag distribution graphs were constructed using makeUCSCfile command and reads from experimental samples were normalized to control or input samples depending on deposited datasets. Logarithmic option -log was used for normalisation. We also selected -fragLength 200 and -inputFragLength 200 options to get data corresponding to single nucleosome.

Tag density distribution plots were visualized using R studio software (R version 3.5.2 "Eggshell Igloo").

\section{Dot-blot and hybridisation}

Dot blot was performed according to previously described protocol (Mozgova et al, 2010). All immunoprecipitated DNA was alkali-transferred onto a Hybond-N+ nylon membrane (Amersham) and hybridized with $\alpha-{ }^{32} \mathrm{P}$ labelled 18S rDNA probe (Mozgova et al, 2010). Signals were visualized using Fujifilm FLA7000 system. Signal intensity was analysed in Fujifilm Multi Gauge software and normalized to input signals. We used following primers for probe labelling. Probes for transcriptional start site and $18 \mathrm{~S}$ were prepared using TSS rDNA Fwd: (AGTATCCTTATGATGCATGCCA), TSS rDNA Rev: (CCCTAACGCCTCGAAGAACTAAT

), 18S rDNA Fwd: (CTAGAGCTAATACGTGCAACAAAC) and $18 \mathrm{~S}$ rDNA Rev: (GAATCGAACCCTAATTCTCCG) with plant genomic DNA as a template according to (Mozgova et al, 2010).

\section{Microscopy and image analysis}

Whole-mount FISH, chromatin and DNA fiber images were acquired on an upright epifluorescence microscope Zeiss AxioImager Z2 using a 63x (1.40 NA) Plan Apochromat or a 100x (1.40 NA) Plan Apochromat objective, using appropriate filters. Images were captured on a Hamamatsu ORCA Flash camera with $2048 \times 2048$ pixel resolution. 3D-deconvolution on whole-mount FISH samples was conducted in ZEN software, using the slow iterative deconvolution algorithm. 


\section{FLIM-FRET}

Fluorescence resonance energy transfer (FRET) was evaluated using fluorescence lifetime imaging (FLIM) and performed on 10-d-old A.thaliana seedlings using the following lines: RedGreen, GreenRed, HTR3-GFP (control) and HTR4-GFP (control). Seedlings were transferred from growth plates onto microscopic slides and imaged using Zeiss LSM 780 (Carl Zeiss MicroImaging GmbH) microscope equipped with "In Tune" laser (488 nm for GFP, $561 \mathrm{~nm}$ for mRFP). FLIM was performed at $488 \mathrm{~nm}$ and $40 \mathrm{MHz}$ frequency and recorded using HPM 100-40 (Becker\&Hickl, Berlin, Germany) hybrid detector, SPC-150 TCSPC (Becker\&Hickl) module and SPCM64 software. Lifetime images were further processed and analyzed in SPCImage 6.4 software (Becker\&Hickl). FRET levels were further calculated as [fluorescence lifetime(control) - fluorescence lifetime(experiment)]/fluorescence lifetime(control). Acceptor over donor ratio was calculated as [fluorescence intensity(acceptor) - fluorescence intensity (donor)]/fluorescence intensity(acceptor). Fluorescence intensity was measured with Fiji software using images of studied nuclei taken prior to FLIM measurement. ROI (region of interest) was selected (whole nucleus, nucleolar chromocenters) and mean fluorescence intensity was measured.

\section{STED microscopy}

Two-color STED microscopy was performed on the Abberior Instruments Expert Line STED system equipped with Nikon Eclipse Ti-E microscopy body and Nikon CFI Plan Apo Lambda 60x Oil, NA 1.40 objective. Sample was illuminated with pulsed $561 \mathrm{~nm}$ and $640 \mathrm{~nm}$ lasers and depleted by pulsed $775 \mathrm{~nm}$ STED laser of 2D donut shape formed by spatial light modulator. Fluorescence signal passed the pinhole set to $1 \mathrm{AU}$, was filtered by emission filters $(580-630 \mathrm{~nm}$ and $650-720 \mathrm{~nm})$ and was detected with single photon counting modules (Excelitas Technologies). STED images were scanned with a pixel size of $20 \mathrm{~nm} \times 20 \mathrm{~nm}$, $10 \mu \mathrm{s}$ dwell time, and in line interleaved acquisition with time gated detection using the Imspector software (Abberior Instruments). DAPI was acquired with a $405 \mathrm{~nm}$ laser in a conventional confocal mode. For STED microscopy, rDNA was labelled with Alexa Fluor 594 and Myc-tagged histones were labelled with $\alpha$-mouse Abberior Star Red secondary antibody (1:100 dilution).

Colocalisation analysis was performed in Icy image analysis software in the "Colocalisation studio" plugin(Lagache et al, 2015). For all the samples, regions of interest were selected around rDNA foci $(n=20$ per condition) and Pearson R correlation coefficient and cross-correlation analysis was calculated.

\section{Structured Illumination Microscopy}

Structured illumination microscopy was performed on Nikon 3D N-SIM microscope (inverted Nikon Eclipse Ti-E, Nikon) equipped with a Nikon CFI SR Apo TIRF objective (100x oil, NA 1.49). Structured illumination pattern projected into the sample plane was created on a diffraction grating block (100 EX V-R 3D-SIM) for laser wavelengths 488, 561 and $647 \mathrm{~nm}$. Excitation and emission light was separated by filter cubes with appropriate filter sets SIM488 (ex. 470-490, em. 500-545), SIM561 (556-566, 570-640) and SIM647 (590$650,663-738)$. Emission light was projected through a 2.5x relay lens onto the chip of the EM CCD camera (Andor iXon Ultra DU897, $10 \mathrm{MHz}$ at 14-bit, $512 \times 512$ pixels). Laser intensity, EM gain and camera exposure time were set independently for each excitation wavelength. Intensity of fluorescence signal was held within the linear range of the camera. 15 images ( 3 rotations and 5 phase shifts) were recorded for every plane and color. SIM data were processed in NIS-Elements AR. Before sample measurement, the symmetry of point spread function was checked with $100 \mathrm{~nm}$ red fluorescent beads (580/605, Carboxylate-Modified Microspheres, Life Technologies) mounted in Abberior Liquid Mount medium (Abberior), and optimized by adjusting objective correction collar.

\section{Single Molecule Localisation Microscopy}

SMLM experiments (STORM) were acquired on a N-STORM system (Nikon) equipped with Nikon Eclipse Ti body, objective Nikon CFI HP Apo TIRF 100x Oil/NA 1.49, Perfect Focus System (hardware autofocus for the stabilization of z-position) and EM CCD Andor iXon Ultra DU897 camera. For STORM, $647 \mathrm{~nm}$ laser beam (fiber output power $125 \mathrm{~mW}$ ) was focused by $2 \mathrm{x}$ magnifying lenses in TIRF Illuminator unit to reach higher laser intensity in the sample. The sample was illuminated by highly inclined laser beam to 
improve the signal to noise ratio. Excitation and emission light was separated on 405/488/561/647 nm Laser Quad Band filter cube (TRF89902, Chroma), specifically, the far-red emission was collected in the emission range 674-785 $\mathrm{nm}$. For spectral demixing, the microscope was adapted by insertion of the W-VIEW GEMINI Image splitting optics (A12801-01, Hamamatsu) to the right port of microscope in front of the EM CCD camera. The fluorescence emission was split to shorter and longer wavelengths by $700 \mathrm{~nm}$ dichroic beam splitter (FF700-Di01, Semrock). All emission wavelengths were detected by the EM CCD camera. Sequences of frames (typically 20 000-30 000 frames) were acquired in NIS-Elements software (version 5.11.02) in Fast Time-Lapse mode to capture the images with the frame rate 31.3 FPS. The time series were measured in selected region of interest with the image size of 512x128 pixels and pixel size $107 \mathrm{~nm}$ (the result of combination $100 \mathrm{x} / 1.49$ NA objective, a $1.5 \mathrm{x}$ zoom adapter and the camera pixel size of $16 \times 16 \mu \mathrm{m}^{2}$ ). Before the acquisition, the sample was illuminated by $647 \mathrm{~nm}$ laser to switch off most of the molecules to the dark state. The acquisition was started after detecting single molecules only. The imaging buffer (50 mM Tris$\mathrm{HCl}, 10 \mathrm{mM} \mathrm{NaCl}, 10 \%$ (w/v) glucose, pH 8 with $50 \mathrm{mM} \beta$-mercaptoethylamine (MEA, 30070, SigmaAldrich), $1.1 \mathrm{mg} / \mathrm{ml}$ Glucose Oxidase from Aspergillus niger (G2133, Sigma-Aldrich) and $100 \mu \mathrm{g} / \mathrm{ml}$ Catalase from bovine liver (C40, Sigma-Aldrich) was freshly prepared on ice before imaging. Image reconstruction was performed in rapidSTORM software (Wolter et al, 2012) followed by drift correction based on cross-correlation analysis in ThunderSTORM (Ovesny et al, 2014) . Localisations were filtered by localisation intensity and precision. For two-color images, spectral demixing of AF647 and CF680 was performed in SD-mixer software (Tadeus et al, 2015). Transformations of localisation tables between the used softwares we performed in custom writen Python scripts. 


\section{Supplementary figures}
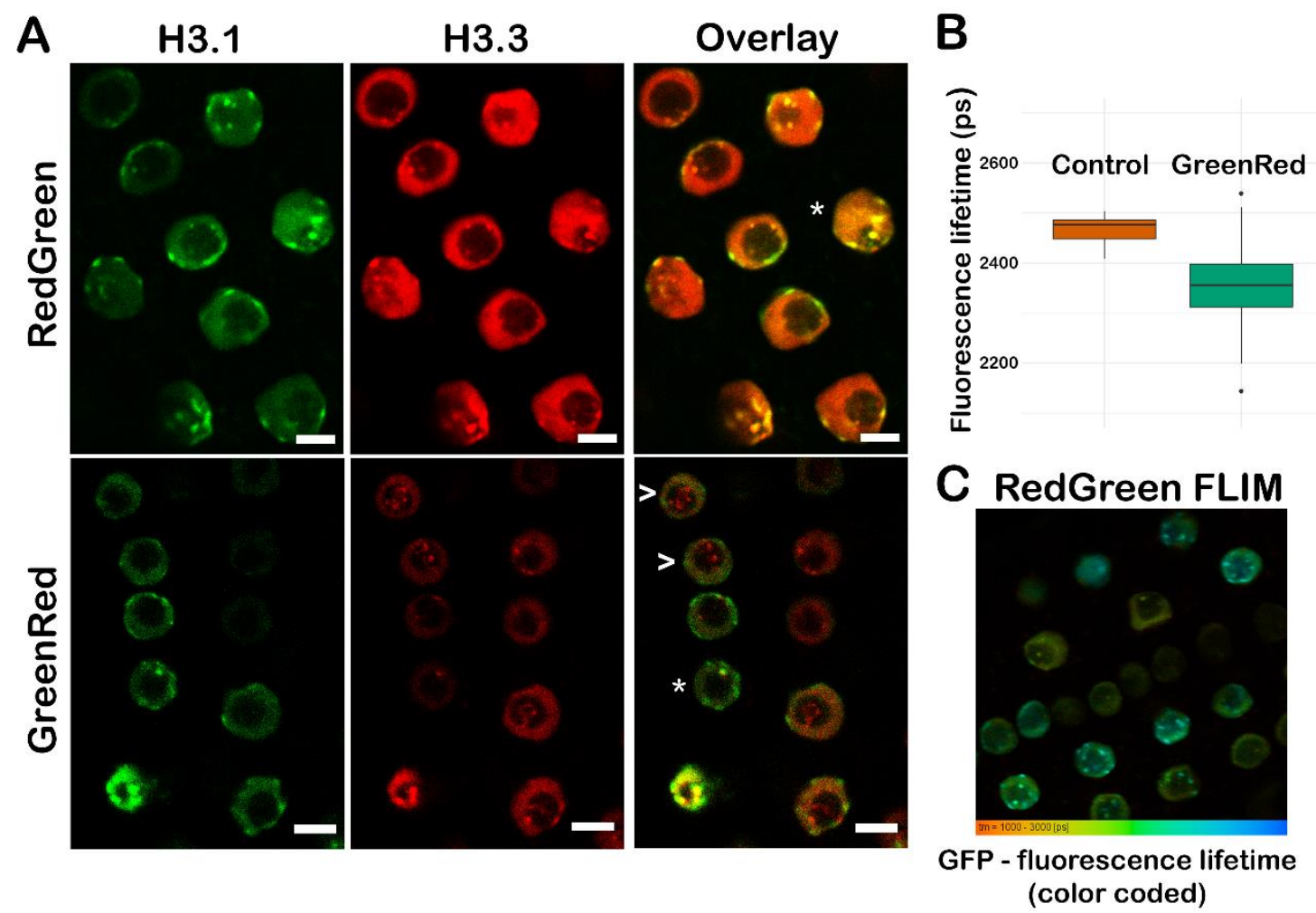

Fig. S1. H3.1 and H3.3 distribution in root tissues

(A) Representative images of H3.1 and H3.3 distribution in root tissue for GreenRed (H3.3-GFP; H3.1mRFP) and RedGreen (H3.3-mRFP; H3.1-GFP) plant lines. H3.1 always color-coded green and H3.3 red for clarity Nuclei with monovariant nucleolar foci are marked by an arrowhead, nuclei with bivariant nucleolar foci are marked by an asterisk. (B) FLIM-FRET experiment showing fluorescence lifetimes $(\tau)$ for control (H3.3-GFP) and GreenRed plant lines. (C) Image of root nuclei showing differences in GFP lifetime in individual nuclei. Fluorescence lifetime is color coded from red (shortest $\tau$ in red, longest $\tau$ in blue). Scale bar $-5 \mu \mathrm{m}$. 


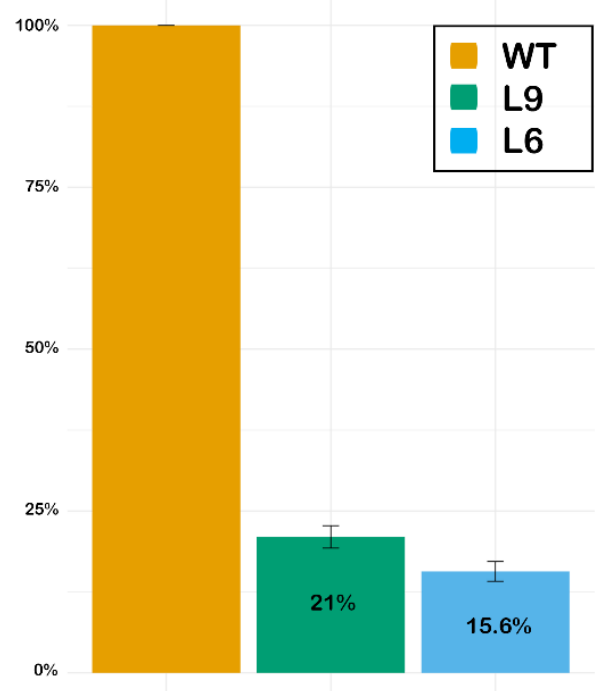

Fig. S2. Relative 45S rDNA copy number in seedlings of WT and low rDNA copy plants (L6, L9).

Relative $45 \mathrm{~S}$ rDNA copy number of low copy lines was evaluated by qPCR in three replicas. WT sample was considered as reference for calculations. 


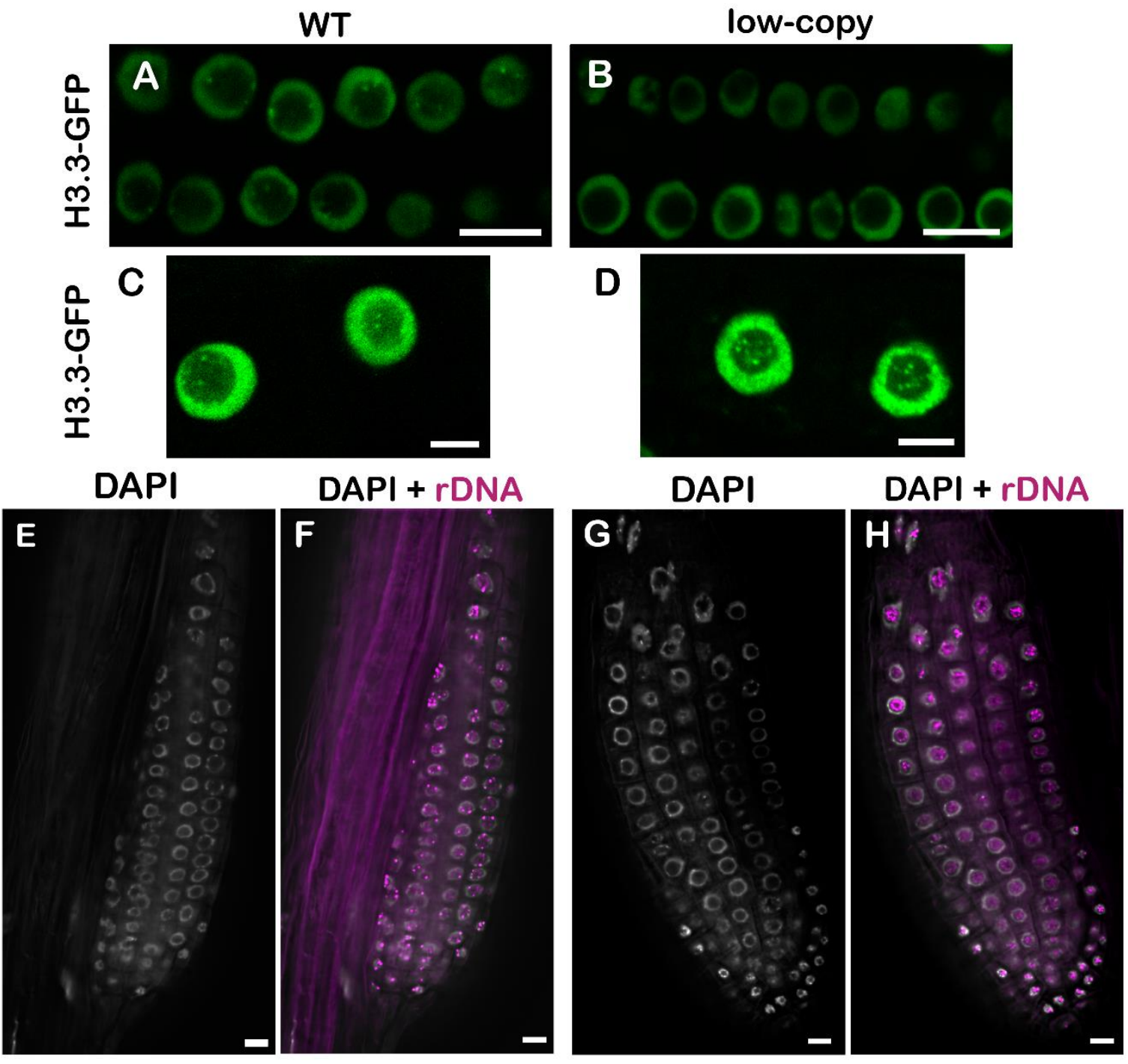

Fig. S3. Loss of rDNA chromocenters in low-copy plants

(A and B) Distribution of H3.3-GFP in actively dividing nuclei of (A) WT and (B) low copy roots (L9 plant line), confocal images. (C and D) Examples of nuclei from the transition zone with $\mathrm{H} 3.3$ foci from both (C) WT and (D) low-copy line L9. (E-H) Organization of rDNA in lateral roots of (F) WT and (H) low copy plants (L6 plant line), rDNA (magenta), DAPI (grey, images E and G), wide-field microscopy combined with deconvolution. Note the absence of perinucleolar rDNA foci in the low-copy plant line $(\mathrm{H})$. Scale bar $-5 \mu \mathrm{m}$ (A and $\mathrm{B}) ; 10 \mu \mathrm{m}(\mathrm{C}-\mathrm{H})$. 

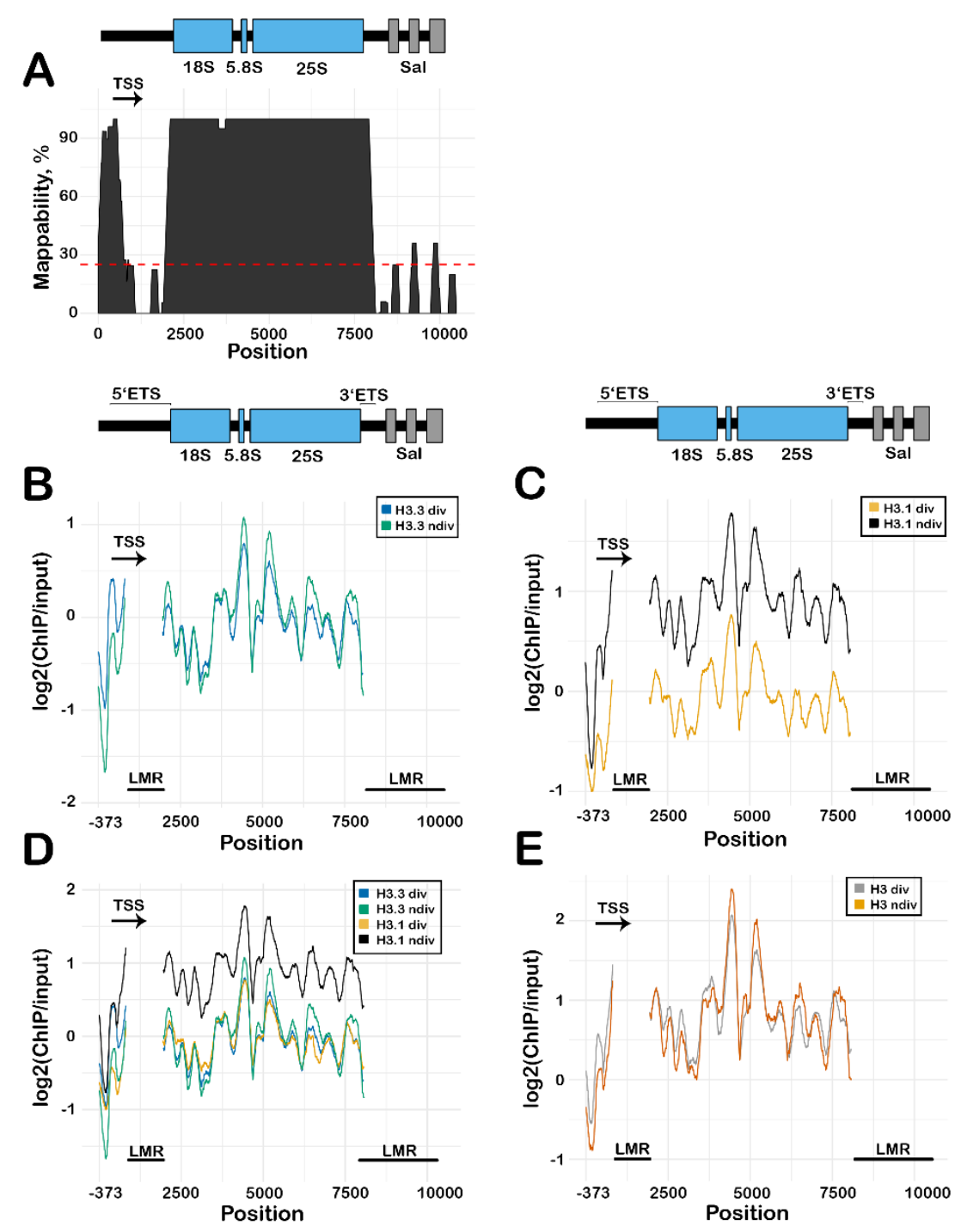

Fig. S4. ChIP-seq read mappability and histone variant distribution in different tissues

(A) Schematic depiction of rDNA unit with graph showing the mappability of the rDNA sequence. Regions below the red line show less than 25\% mappability and are considered unreliable for further analysis (labelled as LMR at further images). Distribution profiles (B) of H3.3 in dividing (H3.3 div) and non-dividing tissue (H3.3 ndiv); (C) H3.1 in dividing (H3.1 div) and non-dividing tissue (H3.1 ndiv); (D) H3.1 and H3.3 distribution profiles in dividing (H3.1 div, H3.3 div) and non-dividing tissue (H3.1ndiv, H3.3 ndiv); (E) H3 profile in dividing (H3 div) and non-dividing (H3 ndiv) tissue. Publicly available ChIP-seq datasets were used; ETS - external transcribed spacer; LMR - low mappability region; TSS - transcriptional start site. 

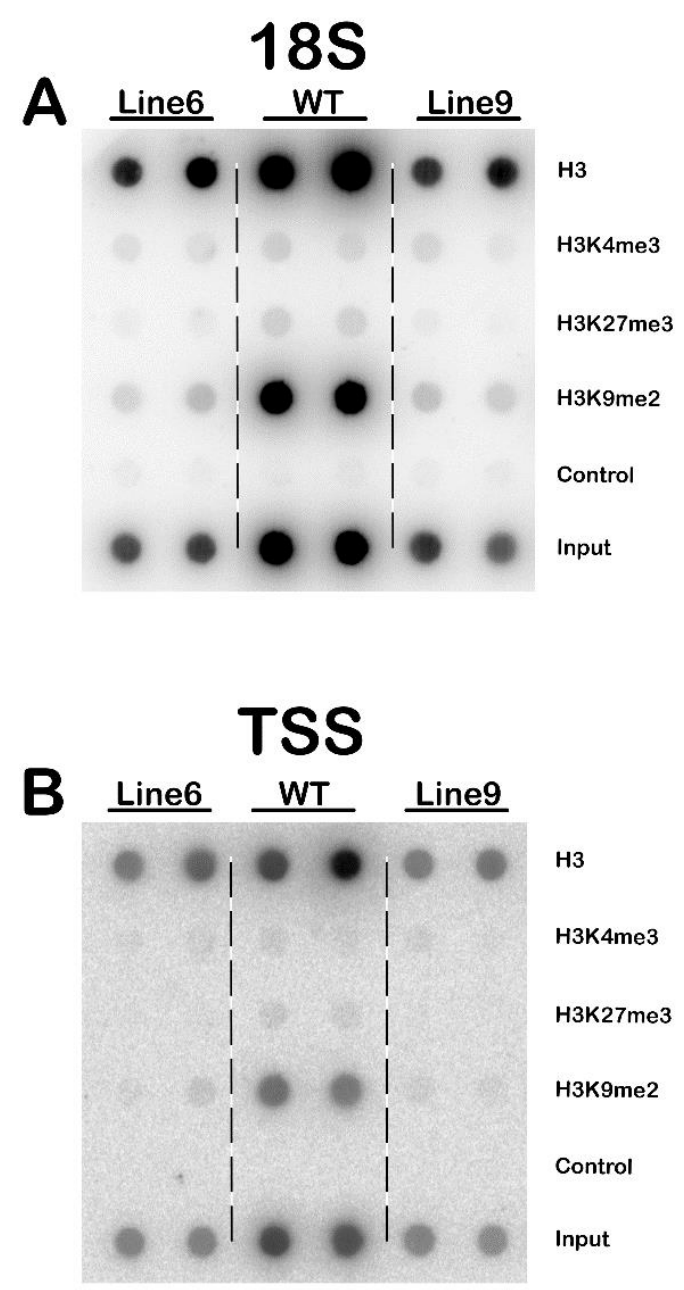

Fig. S5. Analysis of histone modifications with ChIP and subsequent Dot-blot (long exposure)

ChIP experiments with subsequent dot-blot signal detection showing levels of $\mathrm{H} 3$ histone and corresponding epigenetic marks at the (A) $18 \mathrm{~S}$ and (B) TSS regions of a rDNA unit. Membranes were overexposed in order to visualize the weaker signals coming from some of epigenetic modifications. 


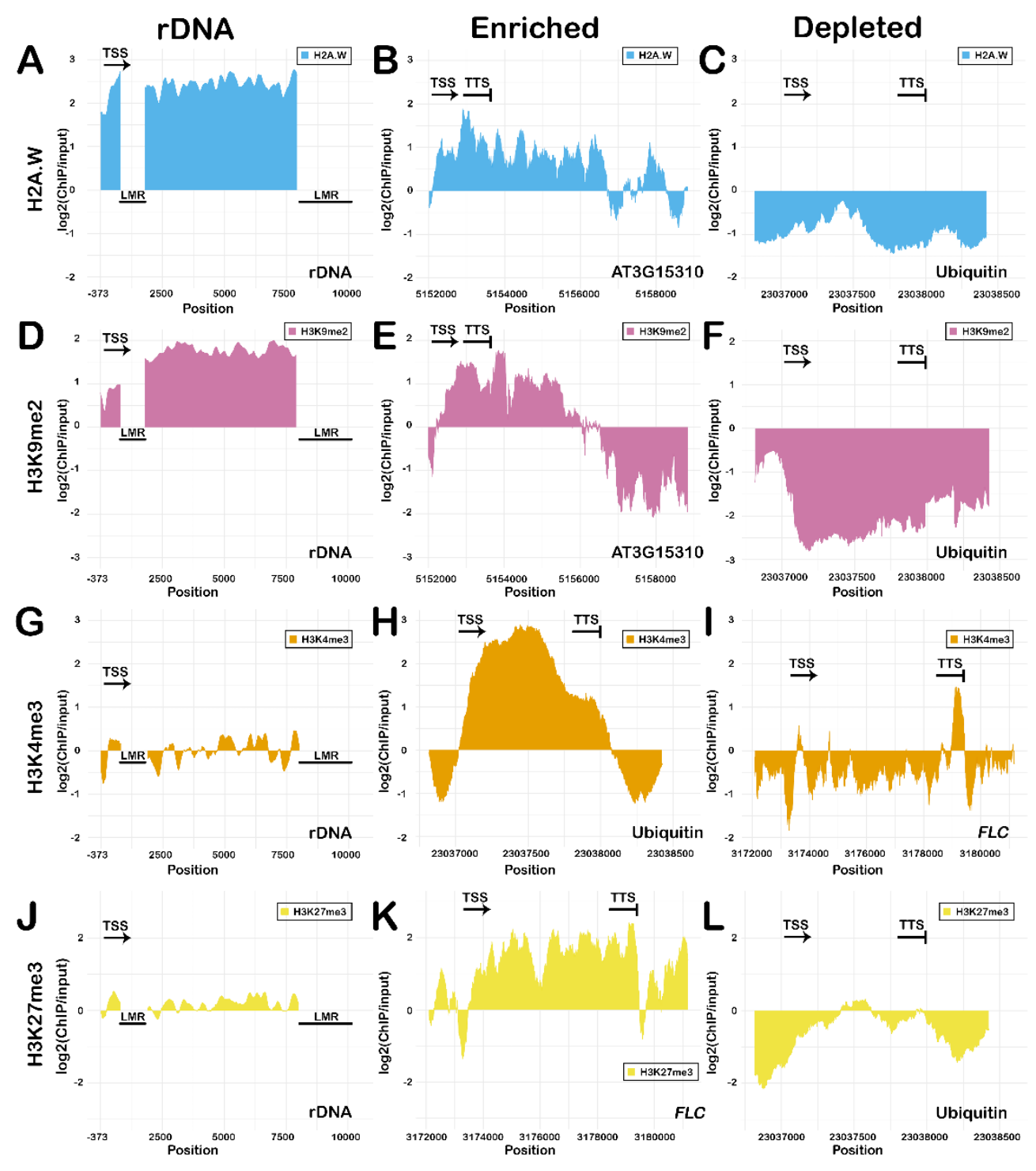

Fig. S6. Comparative analysis ChIP-seq signal from epigenetic marks in rDNA and regions of enrichment or depletion

(A-C) H2AW and (D-F) H3K9me2 levels at (A and D) rDNA; (B and E) enriched region at AT3G15310 (transposable element); (C and F) depleted region at UBIQUITIN 5 gene. G-I) H3K4me3 levels at (G) rDNA; (H) enriched region at UBIQUITIN 5 gene; (I) depleted region at Flowering Locus C (FLC). (J-L) H3K27me3 levels at (J) rDNA; (K) enriched region at $F L C$; (L) depleted region at ubiquitinin5 gene. LMR - low mappability region, TSS - transcriptional start site, TTS - transcriptional termination site. 


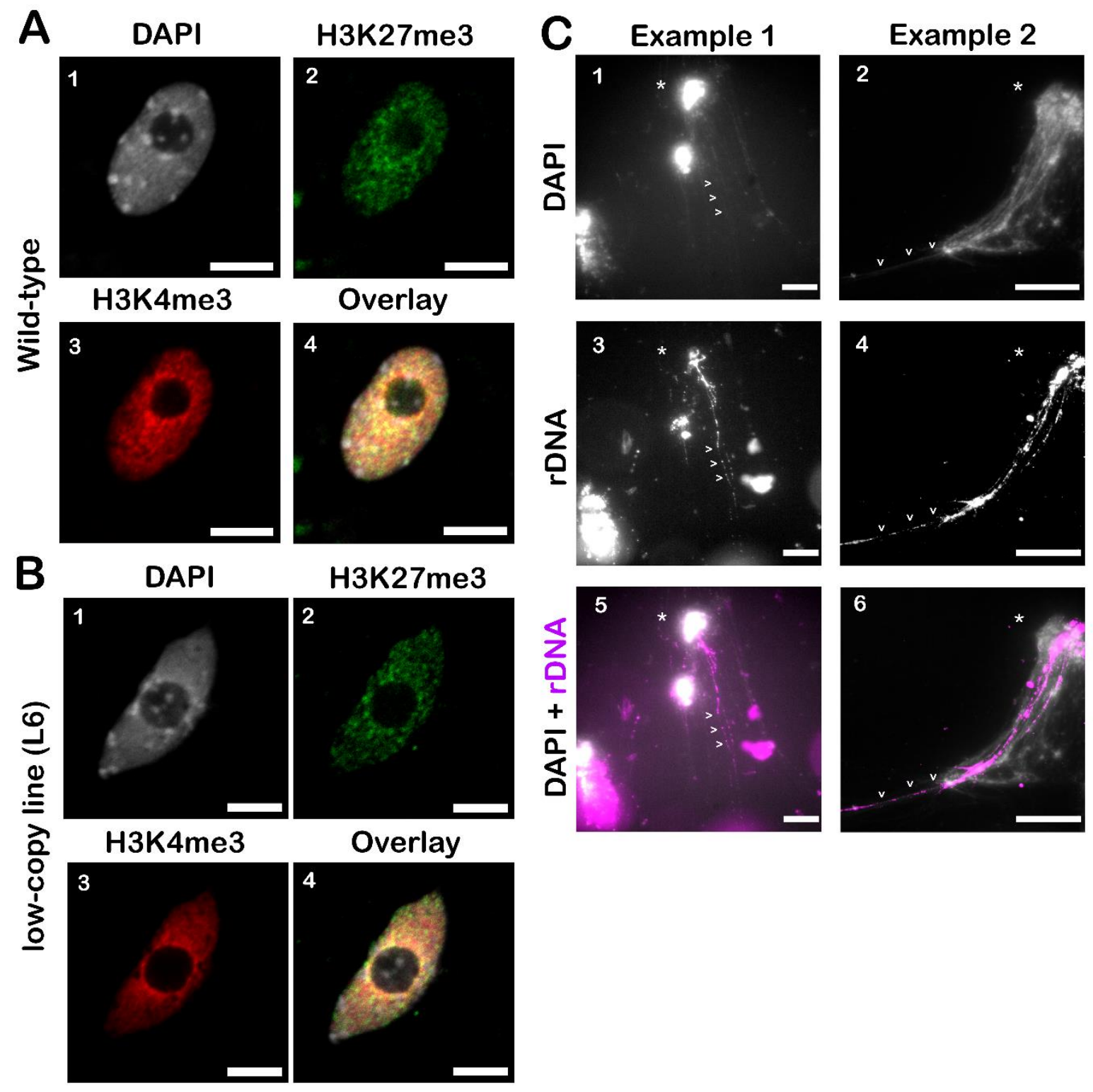

Fig. S7. Visualization of histone marks in isolated nuclei and chromatin fiber FISH validation

(A) Distribution of histone marks (2) H3K27me3 and (3) H3K4me3 in nuclei of WT plants using confocal microscopy. (B) Analysis of histone marks (2) H3K27me3 and (3) H3K4me3 in low-copy plant line L6. (C) FISH on chromatin fibers targeting rDNA. (1-2) Chromatin fibers extending from partially lysed nuclei seen in DAPI. (3-6) Hybridisation of rDNA probe to some of some of the fibers extending from lysed nuclei. Partially lysed nuclei are marked by an asterisk. Chromatin fibers corresponding to rDNA clusters are indicated by arrowheads. Scale bar $-5 \mu \mathrm{m}(\mathrm{A}, \mathrm{B}) ; 10 \mu \mathrm{m}(\mathrm{C})$. 
rDNA

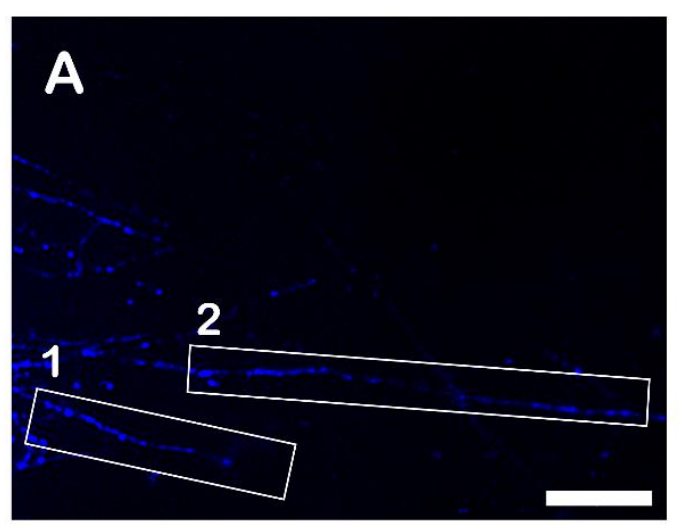

H3K4me3

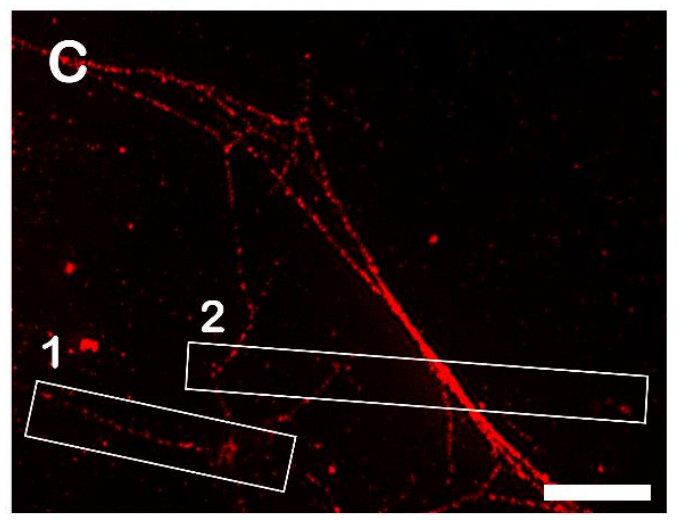

H3K9me2

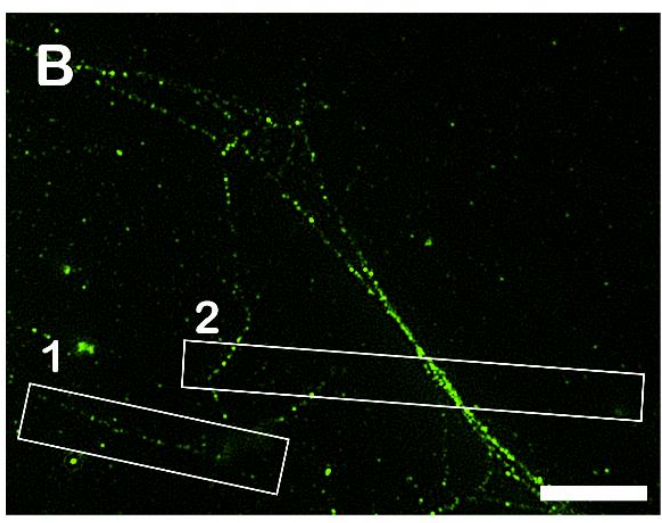

Overlay

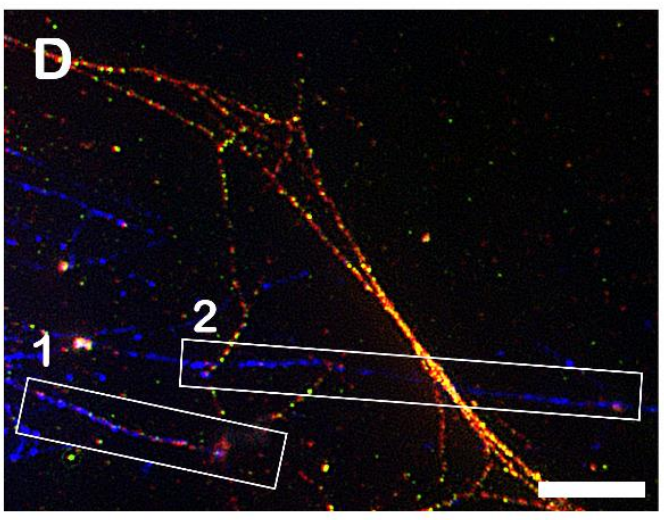

Fig. S8. Detection of histone modifications in chromatin fibers (SIM-microscopy)

(A) Signal from the rDNA probe marks several fibers in the field of view (FOV). Some are decorated with histone marks (rectangle 1) and some harbor nucleosome-free regions (rectangle 2). (B and C) Distribution of the repressive histone mark $\mathrm{H} 3 \mathrm{~K} 9 \mathrm{me} 2(\mathrm{~B})$ and activating histone mark $\mathrm{H} 3 \mathrm{~K} 4 \mathrm{me} 3(\mathrm{C})$ on chromatin fibers. D) Overlay of the channels. Note that the absence of histone marks in rectangle 2 is not due to "histone stripping" during fiber extension, since other fibers in the FOV show strong signals from histone marks. Scale bar $-5 \mu \mathrm{m}$. 


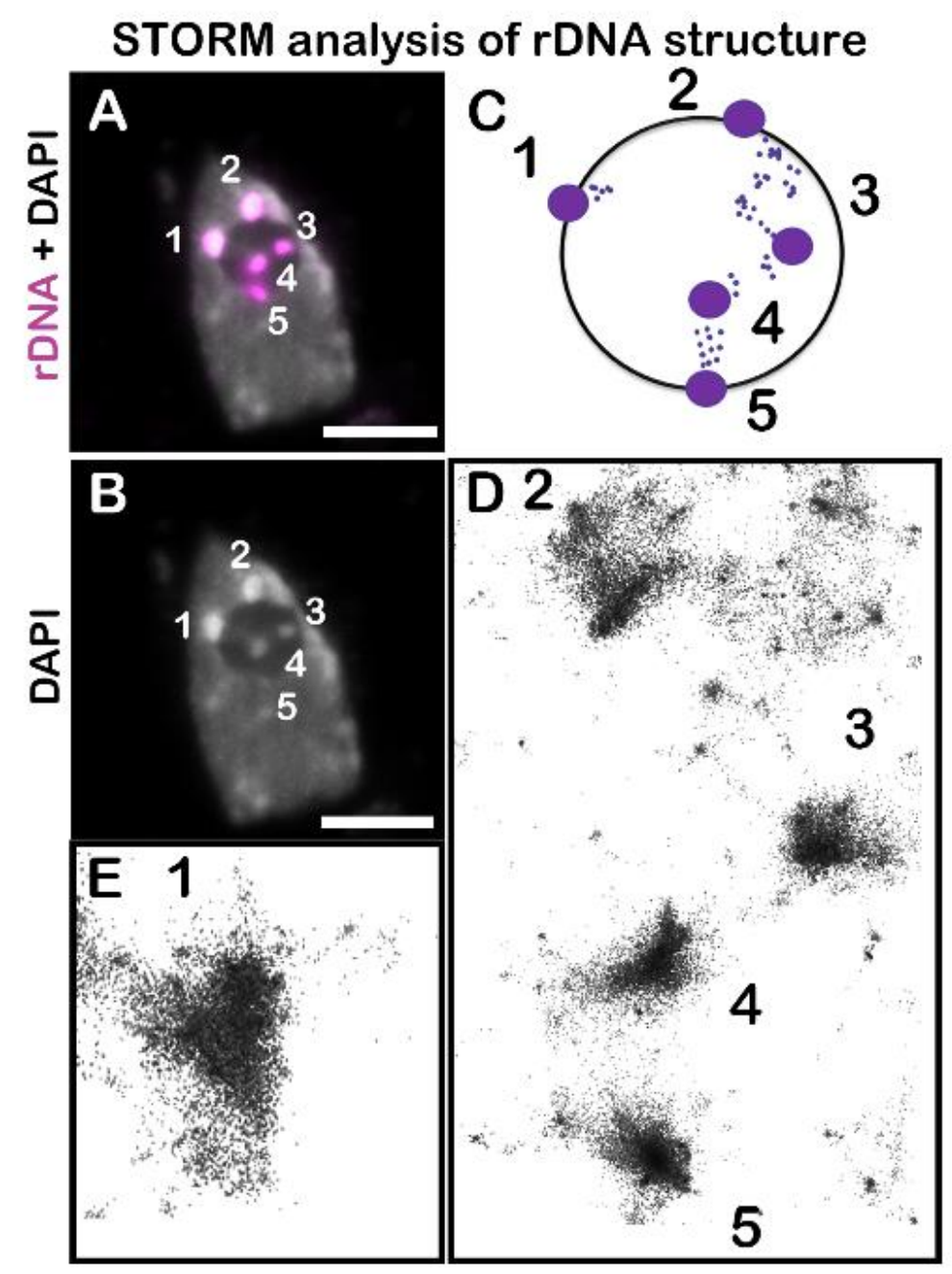

Fig. S9. Ultrastructure of rDNA revealed by dSTORM.

(A and B) Widefield image of a nucleus hybridized with a rDNA probe. Individual perinucleolar chromocenters (PNCs; 1,2,5) and nucleolar foci (NF; 3,4) are depicted schematically in (C). Some PNCs do not appear to extend into the nucleolus (E, 1), while rDNA signals between PNCs and NF can be seen in D (chromocenters 2-3; 4-5), suggesting possible connections. Scale bar $-5 \mu \mathrm{m}$. 


\section{Supplementary references}

Dvorackova M, Raposo B, Matula P, Fuchs J, Schubert V, Peska V, Desvoyes B, Gutierrez C, Fajkus J (2018) Replication of ribosomal DNA in Arabidopsis occurs both inside and outside the nucleolus during S phase progression. J Cell Sci 131

Exner V, Taranto P, Schonrock N, Gruissem W, Hennig L (2006) Chromatin assembly factor CAF-1 is required for cellular differentiation during plant development. Development 133: 41634172

Galbraith DW, Janda J, Lambert GM (2011) Multiparametric analysis, sorting, and transcriptional profiling of plant protoplasts and nuclei according to cell type. Methods Mol Biol 699: 407-429

Havlova K, Dvorackova M, Peiro R, Abia D, Mozgova I, Vansacova L, Gutierrez C, Fajkus J (2016) Variation of 45S rDNA intergenic spacers in Arabidopsis thaliana. Plant Mol Biol 92: 457-471

Heinz S, Benner C, Spann N, Bertolino E, Lin YC, Laslo P, Cheng JX, Murre C, Singh H, Glass CK (2010) Simple combinations of lineage-determining transcription factors prime cis-regulatory elements required for macrophage and B cell identities. Molecular cell 38: 576-589

Cheung MS, Down TA, Latorre I, Ahringer J (2011) Systematic bias in high-throughput sequencing data and its correction by BEADS. Nucleic acids research 39: e103

Lagache T, Sauvonnet N, Danglot L, Olivo-Marin JC (2015) Statistical analysis of molecule colocalization in bioimaging. Cytometry Part A : the journal of the International Society for Analytical Cytology 87: 568-579

Liang YM, Wang X, Ramalingam R, So KY, Lam YW, Li ZF (2012) Novel nucleolar isolation method reveals rapid response of human nucleolar proteomes to serum stimulation. J Proteomics 77: $521-530$

Mandakova T, Lysak MA (2008) Chromosomal phylogeny and karyotype evolution in $\mathrm{x}=7$ crucifer species (Brassicaceae). The Plant cell 20: 2559-2570

Mozgova I, Mokros P, Fajkus J (2010) Dysfunction of chromatin assembly factor 1 induces shortening of telomeres and loss of 45S rDNA in Arabidopsis thaliana. Plant Cell 22: 2768-2780

Otero S, Desvoyes B, Peiro R, Gutierrez C (2016) Histone H3 Dynamics Reveal Domains with Distinct Proliferation Potential in the Arabidopsis Root. Plant Cell 28: 1361-1371

Ovesny M, Krizek P, Borkovec J, Svindrych Z, Hagen GM (2014) ThunderSTORM: a comprehensive ImageJ plug-in for PALM and STORM data analysis and super-resolution imaging. Bioinformatics 30: 2389-2390

Pavlistova V, Dvorackova M, Jez M, Mozgova I, Mokros P, Fajkus J (2016) Phenotypic reversion in fas mutants of Arabidopsis thaliana by reintroduction of FAS genes: variable recovery of telomeres with major spatial rearrangements and transcriptional reprogramming of 45S rDNA genes. Plant $J$ 88: 411-424 
Stroud H, Otero S, Desvoyes B, Ramirez-Parra E, Jacobsen SE, Gutierrez C (2012) Genome-wide analysis of histone H3.1 and H3.3 variants in Arabidopsis thaliana. Proceedings of the National Academy of Sciences of the United States of America 109: 5370-5375

Tadeus G, Lampe A, Schmoranzer J (2015) SDmixer-a versatile software tool for spectral demixing of multicolor single molecule localization data. Methods and applications in fluorescence 3: 037001

Wolter S, Loschberger A, Holm T, Aufmkolk S, Dabauvalle MC, van de Linde S, Sauer M (2012) rapidSTORM: accurate, fast open-source software for localization microscopy. Nature methods 9: 1040-1041

Zentner GE, Henikoff S (2014) High-resolution digital profiling of the epigenome. Nature reviews Genetics 15: 814-827 